\title{
Scale effect analysis of early-termination fixed-complexity sphere detector
}

\author{
Rongrong Qian ${ }^{1}$, Yuan $\mathrm{Qi}^{2}{ }^{*}$, Tao Peng ${ }^{3}$, Jun Yang ${ }^{1}$ and Wenbo Wang ${ }^{3}$
}

\begin{abstract}
In this paper, we show that the early-termination fixed-complexity sphere detector (ET-FSD) has a scale effect when being employed in multi-antenna systems that have to detect signals of multiple users under the constraint of sum complexity. The so-called scale effect stands for a kind of effect in which the complexity per user can be reduced as the number of users increases without loss of diversity order. The main contributions of this study are as follows: (1) we establish a mathematical model based on the large deviation principle to apply the concept of scale effect to ET-FSD; (2) we prove the existence of the scale effect of ET-FSD in a multi-user scenario; and (3) we demonstrate that within multi-user systems, the scale effect can be exploited to realize ET-FSD that has the optimal performance in the point view of diversity order under polynomial complexity constraint per user.
\end{abstract}

\section{Introduction}

\subsection{Research on MIMO detection}

Consider a spatial-multiplexing multiple-input multipleoutput (MIMO) system with $n_{\mathrm{T}}$ transmit and $n_{\mathrm{R}}$ receive antennas, with the channel represented by the matrix $\mathbf{H}=$ $\left[\begin{array}{llll}\mathbf{h}_{1} & \mathbf{h}_{2} & \cdots & \mathbf{h}_{n_{\mathrm{T}}}\end{array}\right] \in \mathbb{C}^{n_{\mathrm{R}} \times n_{\mathrm{T}}}$, where $\mathbf{h}_{i}$ is the $i$ th column of $\mathbf{H}$. All entries of the channel matrix $\mathbf{H}$ are independent and identically distributed (i.i.d.) zero-mean complex Gaussian random variables with unit variance. Let the entries of the vector $\mathbf{s}=\left[\begin{array}{lllll}s_{1} & s_{2} & \cdots & s_{n_{\mathrm{T}}}\end{array}\right]^{\mathrm{T}} \in \mathbb{C}^{n_{\mathrm{T}} \times 1}$ be the transmit symbols, with each $s_{i}$ belonging to the constellation set $\mathcal{D}$ with size $m$. The signal model of the MIMO system is given by

$$
\mathbf{y}=\mathbf{H s}+\mathbf{v}=\sum_{i=1}^{n_{\mathrm{T}}} \mathbf{h}_{i} s_{i}+\mathbf{v},
$$

where $\mathbf{y}$ is the received signal vector, and $\mathbf{v}$ is the zeromean circularly symmetric complex Gaussian noise with covariance matrix $\mathcal{E}\left(\mathbf{v} \mathbf{v}^{H}\right)=\sigma^{2} \mathbf{I}_{n_{\mathrm{R}}, n_{\mathbf{R}}}$. We suppose that $\mathbf{H}$ is perfectly known at the receiver. Let $E_{S}$ denote the energy at the transmitter per symbol period, i.e., $E_{s}=\mathcal{E}\left(\left|s_{i}\right|^{2}\right)$.

\footnotetext{
*Correspondence: qiyuan@bupt.edu.cn

2Electronic School, Beijing University of Posts and Telecommunications (BUPT), Beijing 100876, China

Full list of author information is available at the end of the article
}

Without loss of generality, we assume that $E_{s}=1 / n_{\mathrm{T}}$ and $n_{\mathrm{R}} \geq n_{\mathrm{T}}$. Under these conditions, the optimal scheme of MIMO detection that minimizes the average error probability is the maximum-likelihood (ML) detector expressed by

$$
\widetilde{\mathbf{s}}_{\mathrm{ML}}=\arg \min _{\widetilde{\mathbf{s}} \in \mathcal{D}^{n} \mathrm{~T}}\|\mathbf{y}-\mathbf{H} \widetilde{\mathbf{s}}\|_{F}^{2}
$$

In the context of high signal/noise ratio (SNR), the optimality of the ML detector can be interpreted as the full diversity which means that the detector is capable of achieving the diversity order $n_{\mathrm{R}}$ [1]. Despite the optimal performance, however, the ML detector is not a promising scheme of MIMO detection in practice because of its extremely high complexity. It is evident from (1) that the ML detector has to perform an exhaustive search of $m^{n_{\mathrm{T}}}$ possible candidates and, thus, has the exponential complexity ${ }^{\mathrm{a}}$ $C_{\mathrm{ML}}=O\left(m^{n_{\mathrm{T}}}\right)$.

Ever since MIMO techniques became widely accepted as among the few emerging key technologies for wireless communications, realizing the performance of the optimal or near-optimal MIMO detection without exhaustive search has been an interesting topic for decades [2-9]. It is known from [2-7] that the problem of Equation 1 can be formulated as a lattice search problem [2-4], an integerleast-squares problem [5], a semidefinite relaxation prob-

\section{Springer}

(c) 2013 Qian et al.; licensee Springer. This is an Open Access article distributed under the terms of the Creative Commons

Attribution License (http://creativecommons.org/licenses/by/2.0), which permits unrestricted use, distribution, and reproduction in any medium, provided the original work is properly cited. 
lem [6], and a shortest path problem [7] which are shown to have their own efficient solution methods (algorithms). Many low-complexity strategies can also be used to restrict computational complexity or make the search more efficient, including restricting the search or invoking early termination. Even in recent years, MIMO detection has remained an attractive topic [10-15].

A lot of prior works have been done. However, to the authors' knowledge, it is still hard to realize the MIMO detector with optimal performance (regarding both error probability and diversity order) with the polynomial complexity constraint ${ }^{\mathrm{b}}$. It is shown in [5] that, for integer-least-squares problem with small problem size, the expected complexity of the sphere decoder (SD) at high SNR can be approximated by a polynomial function. The closet-lattice search and shortest path algorithm can efficiently cut down the complexity of solving the integer-least-squares problem [4,7]. However, there does not exist a polynomial upper bound on SD complexity [16], closet-lattice search, or shortest path algorithm for any fixed SNR. With some fixed performance degradation (14) [16], the SD has an exponential complexity (in both the worst and the average cases) of $C_{\mathrm{SD}}=$ $O\left(m^{\gamma n_{\mathrm{T}}}\right)$ with $\gamma \in(0,1]$ [16]. The fixed-complexity sphere decoder (FSD) is proved to maintain full diversity with a complexity of $C_{\mathrm{FSD}}=O\left(m^{\sqrt{n_{\mathrm{T}}}}\right)$ that is 'subexponential' [17]. On the other hand, the linear receivers, e.g., zero-forcing (ZF) and minimum mean square error schemes, have low complexity but quite poor diversity order $n_{\mathrm{R}}-n_{\mathrm{T}}+1$ [1]. The situation is discouraging as several profound studies show that many MIMO detectors have their own inherent limitations $[16,18,19]$. Under this situation, the authors believe that exploiting more potential positive factors in MIMO detectors might be one of the appropriate ideas to make the next breakthrough. In this paper, we focus on exploiting the potential positive factor of MIMO detection in a multi-user scenario.

\subsection{MIMO detection in a multi-user scenario}

It can be found that most studies on the complexity of MIMO detection are under a single-user assumption [2-13], while this study focuses on the complexity of MIMO detection in a multi-user scenario. To understand the motivation behind, one can think of the factor that MIMO receivers in base stations would have to detect signals from multiple-access users. In previous studies $[20,21]$, the scale effect-oriented MIMO detector (SEOD) was developed for multi-user system, which was also shown to have a kind of effect called the scale effect in which the complexity per user can be reduced as the number of users increases without loss of diversity order. Moreover, from [21], we know that scale effect would play an important role in a cloud base station because the cloud base station is a large-scale multi-user system where the baseband processes of a great number of base stations (each could have a lot of accessed users) are performed together within a virtual base station pool under the limit of overall run-time.

However, a big question left to us is whether the scale effect only exists in SEOD. If the answer is 'yes', it will mean that the scale effect has limited practical meanings since the SEOD is a very special MIMO detection scheme which has been carefully designed. Whereas if other schemes of MIMO detection also have the scale effect, we would expect that the scale effect could become an important feature of MIMO detection in multi-user scenarios.

\subsection{Contributions}

In this study, we focus on investigating the earlytermination fixed-complexity sphere detector (ET-FSD) that is an extended version of FSD by incorporating earlytermination mechanism. The existence of scale effect in ET-FSD is proven (Theorems 2 and 3). Due to the scale effect, the complexity per user of ET-FSD can be kept under a polynomial constraint while maintaining fulldiversity performance (Theorem 3 ). We note that the original FSD has no scale effect; thus, in a multi-user case, it still achieves full diversity with subexponential complexity. This result implies that the scale effect not only exists in SEOD but also in ET-FSD and, thus, encourages us to extend and deepen the studies on scale effect in the future. Moreover, an analytical framework based on the large deviation principle is established to investigatethe scale effect in this paper, while [20] does not provide such a framework.

\subsection{Organization}

The rest of this paper is organized as follows: In Section 2, the ET-FSD scheme is specified, and its underlying mathematical model is presented. In Section 3, we prove that the ET-FSD in a single-user scenario is able to achieve full diversity; meanwhile, the complexity behavior of ET-FSD is studied considering both single-user and multi-user scenarios. With respect to the multi-user scenario, the scale effect of ET-FSD is rigorously defined, which in Section 4 is proven to exist. Consequently, the numerical results are provided in Section 5. We conclude our paper in Section VI.

\section{Early-termination fixed-complexity sphere decoder}

\subsection{ET-FSD scheme}

Among the existing MIMO detectors, the FSD [9] is an attractive scheme; it is highly suitable for hardware 
implementation due to its fixed complexity and parallelcomputing-friendly features. By simply incorporating an early-termination mechanism into the FSD, we can establish the ET-FSD scheme. The main process of the ET-FSD is a constrained tree search through a tree with $n_{\mathrm{T}}$ levels where $m$ branches originate from each node. The whole tree search can be realized in two stages: full expansion (FE) and single expansion (SE) search stages, which can be summarized as follows:

- The first stage is the FE search in which a full search is performed in $p$ levels, expanding all $m$ branches per node. In this stage, the transmit symbol with larger noise amplification appears at an earlier level of the tree. Note that a larger noise amplification can also be understood as a smaller post-processing SNR.

- The second stage is the SE search in which a single search needs to be performed in the remaining $n_{\mathrm{T}}-p$ levels. Analogous in principle to previous studies (such as in $[17,22]$ ), the SE search expands one branch per node followed by the $\mathrm{ZF}$ estimate.

Besides the constrained tree search, the complete realization of the ET-FSD also includes the preprocessing of the channel matrix (as FSD [9,17,22] does), which determines the detection order of the transmit symbols and has two stages as well: the preprocessing for the FE search and that for the SE search. To be specific, the preprocessing of the channel matrix can be expressed by Algorithm 1 using the following notations. $(\cdot)^{\dagger}$ denotes the Moore-Penrose pseudoinverse. FlagET is a flag that determines whether the early termination is enabled in the algorithm or not, i.e., FlagET = True means enabled, otherwise disabled. The function denoted by find $(\mathbf{b} \neq x)$ finds the indices of the elements of the vector $\mathbf{b}$ that do not equal $x$. The preprocessing is realized iteratively, and within the $j$ th iteration, the effective channel matrix $\mathbf{H}^{j}$ is reconstructed by removing some column from $\mathbf{H}^{j-1}$, where the initialization condition is $\mathbf{H}^{0}=\mathbf{H}$. Accordingly, $\mathbf{P}^{j}$ denotes the Moore-Penrose pseudoinverse of $\mathbf{H}^{j}$. Algorithm 1 returns a as the ordered indices of the transmit symbols. The elements of a with indices 1 to $p$ are the indices of the transmit symbols to be detected in the FE search. Then the remaining $n_{\mathrm{T}}-p$ elements of $\mathbf{a}$ are the indices of the transmit symbols to be detected in the SE search. Furthermore, $\mathbf{b}=\left[b_{1}, b_{2}, \cdots, b_{\left(n_{\mathrm{T}}-j\right)}\right]$ is just an intermediate vector with its elements satisfying $\left\{b_{1}, b_{2}, \cdots, b_{\left(n_{\mathrm{T}}-j\right)}\right\}=\left\{1,2, \cdots, n_{\mathrm{T}}\right\} \backslash\left\{a_{1}, a_{2}, \cdots, a_{j}\right\}$. Also, $\rho \triangleq n_{\mathrm{T}} E_{s} / \sigma^{2}=1 / \sigma^{2}$ is the average SNR at the receiver (a.k.a. the average receive SNR), and $\gamma \in(0,1)$ is a real value.

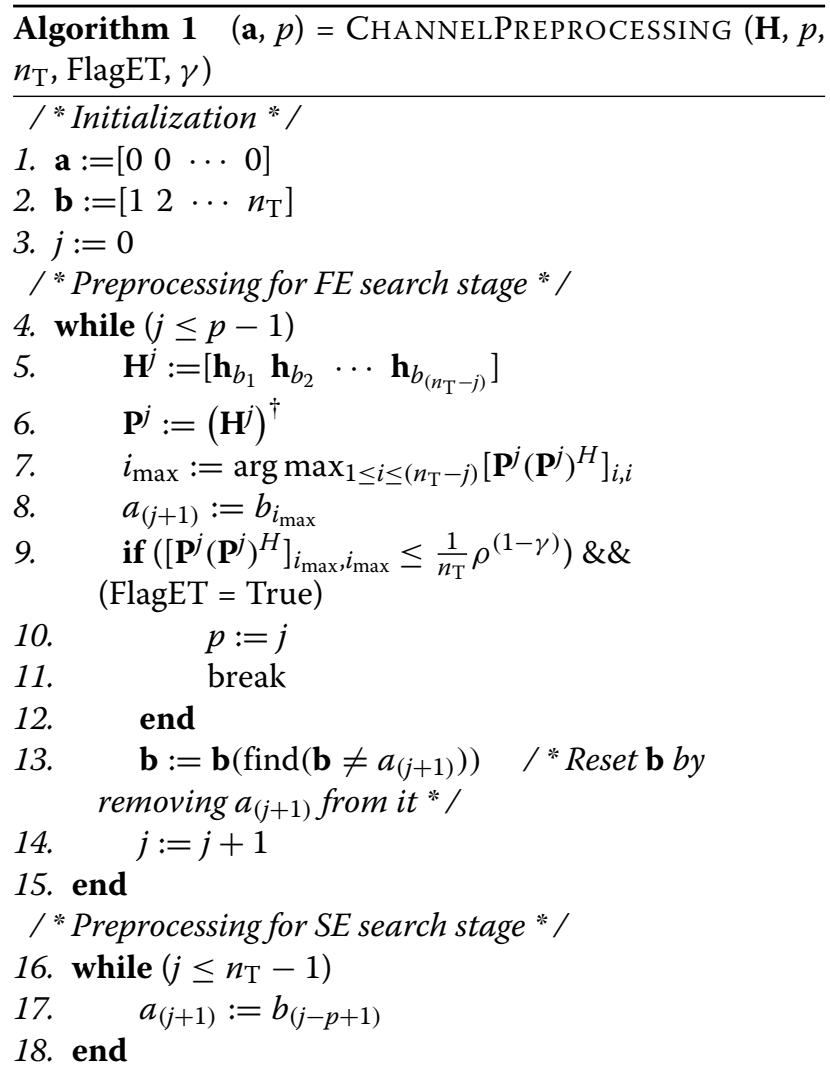

Compared with the channel preprocessing algorithm of the FSD $[9,17,22]$, Algorithm 1 of the ET-FSD has a newly introduced functionality, as seen in lines 9 to 12 , which can terminate early the loop of the preprocessing for the FE search depending on the channel matrix and update $p$ before breaking the loop when FlagET := True is set. Moreover, we have $\bar{p}$ as one input of Algorithm 1 to guarantee full diversity. Early termination implies that the number of levels of the FE search stage could be less than $\bar{p}$. If we set FlagET := False, Algorithm 1 would execute identically as $[17,22]$. Thus, FSD could be considered as a special case of ET-FSD. In line 7, the index of the largest diagonal entry of $\mathbf{P}^{j}\left(\mathbf{P}^{j}\right)^{H}, i_{\text {max }}$, is found. In fact, the diagonal entries of $\mathbf{P}^{j}\left(\mathbf{P}^{j}\right)^{H}$ reflect the post-processing noise amplification (see (5) as well). In line 8 , the original index of the transmit symbol, $a_{(j+1)}$, is obtained based on $i_{\text {max }}$. It is clear that in the $j$ th iteration of channel preprocessing, the $a_{(j+1)}$ th transmit symbol has the largest noise amplification. In this work, for both the convenience of mathematical analysis and the reduction of computational complexity, ZF is employed in the SE stage. Thus, the preprocessing for the SE stage is performed as in line 17.

The constrained tree search of the ET-FSD utilizes a and $p$, which are generated from the preprocessing of the 
channel matrix as the two most significant parameters. Analogous to the FSD, the tree search of the ET-FSD consists of two stages and can be illustrated as in Figure 1. On the other hand, we can express such tree search by Algorithm 2, which is in the iterative fashion. Before the details are examined, the required notation can be given as follows: $\mathcal{D}(k)$ is the $k$ th element of constellation set $\mathcal{D} ; \operatorname{ZF}(\cdot, \cdot)$ denotes the functionality of zeroforcing estimate; $\widetilde{\mathbf{s}}_{\mathrm{SE}} \triangleq\left[\widetilde{s}_{a_{p+1}}, \tilde{s}_{a_{(p+2)}}, \cdots, \widetilde{s}_{a_{n_{\mathrm{T}}}}\right]^{\mathrm{T}} ; \mathbf{H}_{\mathrm{SE}} \triangleq$ $\left[\mathbf{h}_{a_{(p+1)}}, \mathbf{h}_{a_{(p+2)}}, \cdots, \mathbf{h}_{a_{n_{\mathrm{T}}}}\right] ; \mathbf{y}^{j}$ is the intermediate vector in the $j$ th iteration; and $\widetilde{s}_{a_{(j+1)}}$ is the $a_{(j+1)}$ th element of $\widetilde{\mathbf{s}}$. The initial input of Algorithm 2 includes $\mathbf{y}^{0}:=\mathbf{y}, j:=0$, $d_{\max }:=+\infty$ and $\widetilde{\mathbf{s}}:=[0,0, \cdots, 0]$. We note that, similar to most previous works $[9,22]$, the complexity spent on the preprocessing of channel matrix is ignored in our further analysis.

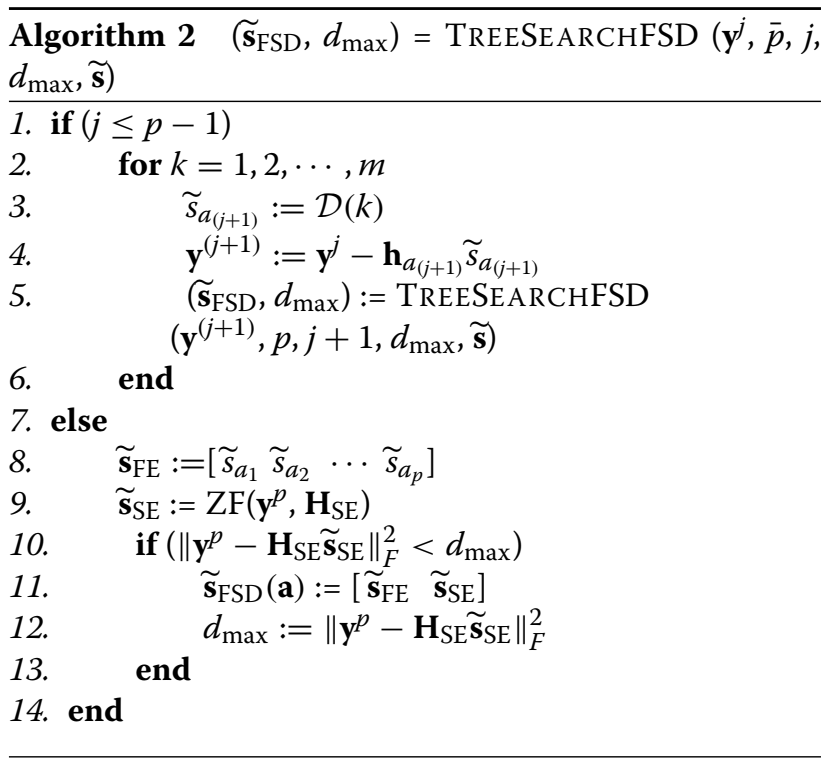

\subsection{Mathematical model of the ET-FSD}

Here we introduce the underlying mathematical model of the ET-FSD. Lines 2 to 6 of Algorithm 2 correspond to the manipulation of the FE search stage, while line 5 recursively invokes TREESEARCHFSD. After the final iteration of the FE search, the signal model becomes

$$
\begin{aligned}
\mathbf{y}^{p}= & \sum_{i \in\left\{a_{(p+1)}, a_{(p+2)}, \cdots, a_{n_{\mathrm{T}}}\right\}} \mathbf{h}_{i} s_{i} \\
+ & \underbrace{\sum_{k \in\left\{a_{1}, a_{2}, \cdots, a_{p}\right\}} \mathbf{h}_{k}\left(s_{k}-\widetilde{s}_{k}\right)+\mathbf{v}}_{\triangleq \mathbf{w}},
\end{aligned}
$$

where $\mathbf{w}$ is the interference caused by the difference between the symbols of $\widetilde{\mathbf{s}}_{\mathrm{FE}}$ and those of the transmit vector. Thus, the signal model left for the SE search is

$$
\begin{aligned}
\mathbf{y}^{p} & =\left[\begin{array}{llll}
\mathbf{h}_{a_{(p+1)}} & \mathbf{h}_{a_{(p+2)}} & \cdots & \mathbf{h}_{a_{n_{\mathrm{T}}}}
\end{array}\right]\left[\begin{array}{c}
s_{a_{(p+1)}} \\
s_{a_{(p+2)}} \\
\vdots \\
s_{a_{n_{\mathrm{T}}}}
\end{array}\right]+\mathbf{w}+\mathbf{v} \\
& =\mathbf{H}_{\mathrm{SE}}\left[\begin{array}{c}
s_{a_{(p+1)}} \\
s_{a_{(p+2)}} \\
\vdots \\
s_{a_{n_{\mathrm{T}}}}
\end{array}\right]+\mathbf{w}+\mathbf{v} .
\end{aligned}
$$

Applying the ZF estimate to (2), $\widetilde{\mathbf{s}}_{\mathrm{SE}}$ would be obtained as $\widetilde{\mathbf{s}}_{\mathrm{SE}}=\left(\mathbf{H}_{\mathrm{SE}}\right)^{\dagger} \mathbf{y}^{p}$.

Herein we consider the path which is perfectly canceled at the FE search and satisfies $s_{k}-\tilde{s}_{k}=0$ for all

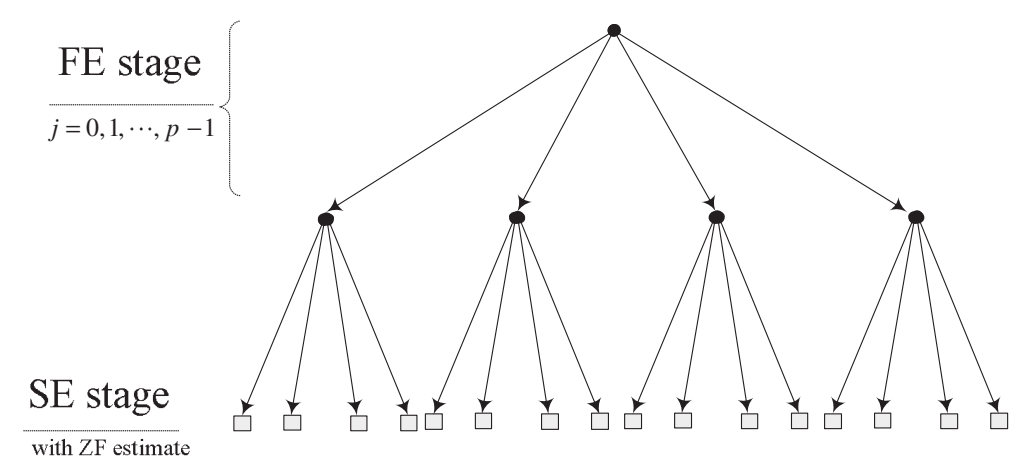

Figure 1 Parallel-computing model of Algorithm 2. It is an example that has $n_{T}=n_{R}=8$ and $m=4$. The number of levels of the FE stage $p=\left\lceil\sqrt{n_{\top}}\right\rceil-1$. 
$k \in\left\{a_{1}, a_{2}, \cdots, a_{p}\right\}$ such that $\mathbf{w}=[0,0, \cdots, 0]$. We can simplify the signal model left for the SE search as

$$
\mathbf{y}^{p}=\sum_{i \in\left\{a_{(p+1)}, a_{(p+2)}, \cdots, a_{n_{\mathrm{T}}}\right\}} \mathbf{h}_{i} s_{i}+\mathbf{v} .
$$

Under such signal model, it should be clear that after the ZF estimate in the SE stage, $\widetilde{\mathbf{s}}_{\mathrm{SE}}=\mathbf{s}_{\mathrm{SE}}+\left(\mathbf{H}_{\mathrm{SE}}\right)^{\dagger} \mathbf{v}$, where $\mathbf{s}_{\mathrm{SE}}=\left[s_{a_{(p+1)}}, s_{a_{(p+2)}}, \cdots, s_{a_{n_{\mathrm{T}}}}\right]^{\mathrm{T}}$.

Meanwhile, lines 8 to 13 of Algorithm 2 perform the SE search in which $m^{p}$ candidate paths would be equalized by the ZF estimate, while one path with minimum Euclidean distance from $\mathbf{y}$ that corresponds to $\widetilde{\mathbf{s}}_{\mathrm{FSD}}$ is returned as the detected vector. In line 11, a specifies the indices of the elements of $\widetilde{\mathbf{s}}_{\text {FSD }}$.

\subsection{A first look at the complexity of ET-FSD}

Let us keep in mind that the FE search of ET-FSD has $p$ levels, where $p$ is determined by Algorithm 1. By referring to $[17,22]$, we know that the complexity of the SE search in ET-FSD does not dominate the overall complexity, but the FE search does. Thus, the overall complexity of the ET-FSD is dominated by $p$ as $\zeta_{p}=O\left(m^{p}\right)$, where $\zeta_{p}$, for the convenience of expression, is defined as the overall complexity of the ET-FSD when the FE search stage has $p$ levels. Obviously, $\zeta_{p}$ is determined by $p$. We should also be aware that the ET-FSD can maintain full diversity as the ML detector as long as $p \geq \bar{p}$, where $\bar{p}$ is the least integer that satisfies

$$
\left(n_{\mathrm{R}}-n_{\mathrm{T}}\right)(\bar{p}+1)+(\bar{p}+1)^{2} \geq n_{\mathrm{R}}
$$

because it is proven in $[17,22]$ that setting the number of the FE stage to $\bar{p}$ is a sufficient condition for the FSD to achieve full diversity.

\section{ET-FSD in a single-user scenario}

\subsection{Error probability analysis}

The error probability of the proposed ET-FSD with disabled early termination (in this case the ET-FSD is actually identical to the FSD), which in general is defined as the probability of the error event $\widetilde{\mathbf{s}}_{\mathrm{FSD}} \neq \mathbf{s}$ averaged over the channel matrix, the additive noise, and the transmitted codewords, has the relation as (see Equations 4 to 7 of [22]) $p_{e \mathrm{FSD}} \triangleq P\left(\widetilde{\mathbf{s}}_{\mathrm{FSD}} \neq \mathbf{s}\right) \leq p_{e \mathrm{ML}}+p_{e \mathrm{SE}}$, where $p_{e \mathrm{ML}}$ is the error probability of the ML detector and $p_{e S E}$ is the probability of the error event $\widetilde{\mathbf{s}}_{\mathrm{SE}} \neq \mathbf{s}_{\mathrm{SE}}$ [22]. Analogously, with enabled early termination, the error probability of the proposed ET-FSD algorithm is defined and bounded as (see Equations 4 to 7 of [22] as well)

$$
p_{e \mathrm{ET}-\mathrm{FSD}} \triangleq P\left(\widetilde{\mathbf{s}}_{\mathrm{ET}-\mathrm{FSD}} \neq \mathbf{s}\right) \leq p_{e \mathrm{ML}}+p_{e \mathrm{ET}-\mathrm{SE}}
$$

where $p_{e \mathrm{ET}-\mathrm{SE}}$ is the probability of the error event $\widetilde{\mathbf{s}}_{\mathrm{SE}} \neq$ $\mathbf{s}_{\mathrm{SE}}$ with FlagET $=$ True.
Recall from Algorithm 1 that the smallest postprocessing SNR (related to the largest noise amplification) of the transmit symbols in the $j$ th iteration of the preprocessing for the FE search stage, given the effective channel matrix $\mathbf{H}^{j}$, can be written as

$$
\eta_{(j+1)}=\min _{1 \leq i \leq\left(n_{\mathrm{T}}-j\right)} \frac{\rho}{n_{\mathrm{T}}} \frac{1}{\left[\mathbf{P}^{j}\left(\mathbf{P}^{j}\right)^{H}\right]_{i, i}} .
$$

Otherwise, equivalently, we have [23]

$$
\max _{1 \leq i \leq\left(n_{\mathrm{T}}-j\right)}\left[\mathbf{P}^{j}\left(\mathbf{P}^{j}\right)^{H}\right]_{i, i}=\frac{\rho}{n_{\mathrm{T}} \eta_{(j+1)}} .
$$

Similar to the derivations in (32) and (33) of [24], since the largest eigenvalue is not less than any diagonal term of a Hermitian matrix (see Section 5.3.1 of [25]), (5) yields $\eta_{(j+1)} \geq \frac{\rho}{n_{\mathrm{T}}} \frac{1}{\lambda_{\max }\left(\mathbf{P}^{j}\left(\mathbf{P}^{j}\right)^{H}\right)}$, where $\lambda_{\max }(\cdot)$ denotes the maximum eigenvalue of a square matrix. On the other hand, $\mathbf{P}^{j}\left(\mathbf{P}^{j}\right)^{H}=\left(\left(\mathbf{H}^{j}\right)^{H} \mathbf{H}^{j}\right)^{-1}$ such that $\lambda_{\max }\left(\mathbf{P}^{j}\left(\mathbf{P}^{j}\right)^{H}\right)=$ $1 / \lambda_{1}\left(\left(\mathbf{H}^{j}\right)^{H} \mathbf{H}^{j}\right)$, and we can get

$$
\eta_{(j+1)} \geq \frac{\rho}{n_{\mathrm{T}}} \lambda_{1}\left(\left(\mathbf{H}^{j}\right)^{H} \mathbf{H}^{j}\right)
$$

where $\lambda_{k}(\cdot)$ is the $k$ th smallest eigenvalue of the matrix.

According to Algorithm 1 and (6), early termination occurs when

$$
\frac{\rho}{n_{\mathrm{T}} \eta_{(j+1)}} \leq \frac{1}{n_{\mathrm{T}}} \rho^{(1-\gamma)},
$$

with $0<\gamma<1$. It then means that the event $\eta_{(j+1)} \geq$ $\rho^{\gamma}$ is identical to the early-termination event. We will see later why the judging criterion for early termination is set as (8). Given (7), the probability of no early termination satisfies

$$
\begin{aligned}
P\left(\eta_{(j+1)}\right. & \left.<\rho^{\gamma}\right) \leq P\left(\lambda_{1}\left(\left(\mathbf{H}^{j}\right)^{H} \mathbf{H}^{j}\right)<n_{\mathrm{T}} \rho^{(\gamma-1)}\right) \\
& \leq P\left(\left(\begin{array}{c}
n_{\mathrm{T}} \\
j
\end{array}\right)^{-1} \lambda_{(j+1)}\left(\mathbf{H}^{H} \mathbf{H}\right)<n_{\mathrm{T}} \rho^{(\gamma-1)}\right) \\
& \leq \beta\left(\left(\begin{array}{c}
n_{\mathrm{T}} \\
j
\end{array}\right) n_{\mathrm{T}} \rho^{(\gamma-1)}\right)^{\left(n_{\mathrm{R}}-n_{\mathrm{T}}\right)(j+1)+(j+1)^{2}},
\end{aligned}
$$

where the second and third inequalities are derived by following Equations 17 and 14 of [22], respectively, and $\beta$ is some real constant according to [26]. 
Let $A_{j}$ be the no early termination event that is identical to the event below

$$
\eta_{(j+1)}<\rho^{\gamma} .
$$

Thus, the complementary event $\bar{A}_{j}$ is equivalent to $\eta_{(j+1)} \geq \rho^{\gamma}$.

Lemma 1. For the event $A_{j}$, it holds that

$$
\lim _{\rho \rightarrow+\infty} P\left(A_{j}\right)=0, \quad \forall j \geq 0 .
$$

Proof. Taking both the relation of (9) and the definition of $A_{j}$ as (10) into consideration, as $\gamma-1<0$, (11) can be obtained directly.

In addition, let $E_{\mathrm{SE}}$ and $E_{\mathrm{ET}-\mathrm{SE}}$ denote the event that $\widetilde{\mathbf{s}}_{\mathrm{SE}} \neq \mathbf{s}_{\mathrm{SE}}$ where $\mathbf{s}_{\mathrm{SE}}$ consists of the symbols of the transmit vector, which are detected in the SE search stage with the early termination disabled and enabled, respectively. Hence, $p_{e \mathrm{SE}}=P\left(E_{\mathrm{SE}}\right)$ and $p_{e \mathrm{ET}-\mathrm{SE}}=P\left(E_{\mathrm{ET}-\mathrm{SE}}\right)$.

Lemma 2. In considering the error probability of the ET-FSD, we can first get $p_{e \mathrm{ET}-\mathrm{SE}} \leq p\left(n_{\mathrm{T}}-\right.$ $p) \bar{N}_{e} \exp \left(-\frac{\rho^{\gamma} d_{\min }^{2}}{4}\right)+p_{\text {eSE. }}$. Thus, recalling from (4), we are further able to obtain $p_{e \mathrm{ET}-\mathrm{FSD}} \leq p\left(n_{\mathrm{T}}-\right.$ $p) \bar{N}_{e} \exp \left(-\frac{\rho^{\gamma} d_{\min }^{2}}{4}\right)+p_{e S E}+p_{e M L}$, where $\bar{N}_{e}$ and $d_{\min }$ are constants that can be found in the proof of this lemma.

Proof. See Appendix 1.

\subsection{Diversity order analysis}

To simplify the expression, evaluation of the performance of MIMO detectors makes use of diversity order, which is a metric constructed from the error probability and average received SNR [27] (an introduction to the diversity order metric and its associated properties can be found in Appendix 2).

Consequently, we define

$$
\begin{aligned}
d_{\mathrm{ET}-\mathrm{FSD}} \triangleq-\lim _{\rho \rightarrow+\infty} \frac{\log p_{e \mathrm{ET}-\mathrm{FSD}}}{\log \rho}, \\
d_{\mathrm{ML}} \triangleq-\lim _{\rho \rightarrow+\infty} \frac{\log p_{e \mathrm{ML}}}{\log \rho}, \\
d_{\mathrm{ET}-\mathrm{SE}} \triangleq-\lim _{\rho \rightarrow+\infty} \frac{\log p_{e \mathrm{ET}-\mathrm{SE}}}{\log \rho},
\end{aligned}
$$

where $d_{\mathrm{ET}-\mathrm{FSD}}$ and $d_{\mathrm{ML}}$ denote the diversity order of the ET-FSD and the ML detector, respectively. Herein (12) can be interpreted as

$$
\begin{aligned}
p_{e \mathrm{ET}-\mathrm{FSD}} & \doteq \rho^{-d_{\mathrm{ET}-\mathrm{FSD}}} \\
p_{e \mathrm{ML}} & \doteq \rho^{-d_{\mathrm{ML}}} \\
p_{e \mathrm{ET}-\mathrm{SE}} & \doteq \rho^{-d_{\mathrm{ET}-\mathrm{SE}}}
\end{aligned}
$$

Based on the above discussion, for the diversity order of the ET-FSD in a single-user scenario, we can obtain the following theorem.

Theorem 1. As the condition of triggering the early termination is appropriately constructed, i.e., $\eta_{(j+1)} \geq \rho^{\gamma}$ with $0<\gamma<1$, the ET-FSD specified by Algorithms 1 and 2 retains full diversity, i.e., $d_{\mathrm{ET}-\mathrm{FSD}}=n_{\mathrm{R}}$.

Proof. See Appendix 3.

\subsection{Complexity behavior}

Recalling from Algorithm 1, one should be aware that the number of FE search of the ET-FSD, $p$, varies with the channel matrix $\mathbf{H}$. Thus, in the reminder of the paper, we will use $p(\mathbf{H})$ to denote $p$ so as to emphasize that $p(\mathbf{H})$ is a function of $\mathbf{H}$. Due to the early termination, the complexity of the ET-FSD, $\zeta_{p(\mathbf{H})}$, is a random variable when the channel matrix is random. For any $p(\mathbf{H})$, it fulfills that $\zeta_{p(\mathbf{H})} \in\left\{\zeta_{0}, \zeta_{1}, \cdots, \zeta_{\bar{p}}\right\}$, where $\zeta_{i}=O\left(m^{i}\right), 0 \leq i \leq \bar{p}$. Thus, $\zeta_{0}=O(1)$. Note that $m^{i}$ implies the number of all possible candidates of $\mathbf{s}$. This complexity measure is the same as in a previous study [17]. We now investigate the properties of $\zeta_{p(\mathbf{H})}$ in terms of the probability and expectation of $\zeta_{p(\mathbf{H})}$.

\section{Lemma 3. There exist the relations}

$$
\lim _{\rho \rightarrow+\infty} P\left(\zeta_{p(\mathbf{H})} \neq \zeta_{0}\right)=\lim _{\rho \rightarrow+\infty} P\left(A_{1}\right)=0,
$$

in particular,

$$
P\left(\zeta_{p(\mathbf{H})} \neq \zeta_{0}\right)=P\left(A_{1}\right) \dot{\leq} \rho^{-(1-\gamma)\left(n_{\mathrm{R}}-n_{\mathrm{T}}+1\right)},
$$

and

$$
\lim _{\rho \rightarrow+\infty} \mathcal{E}\left(\zeta_{p(\mathbf{H})}\right)=\zeta_{0},
$$

where $\dot{\leq}$ is defined similarly as $\doteq$ in Appendix 2 .

\section{Proof. See Appendix 4.}

It is apparent that, given Lemma 3, in the high-SNR regime, the early termination employed by the ET-FSD can efficiently reduce the expected complexity (compared with the FSD) but seems to have no improvement on the worst-case complexity, which is more significant for practical hardware implementation.

\section{Scale effect of ET-FSD}

As a long run concept in microeconomics, 'economies of scale' refers to reductions in unit cost as the size of a facility and the usage levels of other inputs increase [28]. In previous studies $[20,21]$, we proposed extracting the scale effect so as to realize the reductions in complexity per user of SEOD as the number of users increases. In this section, 
we investigate the scale effect of ET-FSD with a large deviation principle-based analytical framework. For notational brevity, we use SCC-ET-FSD to represent the sum complexity constrained ET-FSD in a multi-user scenario.

\subsection{Complexity behavior in a multi-user scenario}

In the point of view of worst-case complexity, this study will show that in a multi-user scenario ${ }^{c}$, the scale effect is one positive factor that should be beneficial to the realization of the ET-FSD (the corresponding analysis can be found in the next subsection). Since the scale effect exists in a multi-user scenario, we now focus on discussing the complexity behavior of the ET-FSD in a multi-user scenario where the MIMO receiver has to detect the signals of $n$ users with the ET-FSD performed for each user.

To simplify the analysis, we assume that different users in a multi-user scenario suffer i.i.d. channel fading and have the same average received SNR. Therefore, if we let the random variable, $\zeta_{p(\mathbf{H}), j}$, denote the complexity of the ET-FSD of the $j$ th user, we will naturally get that $\zeta_{p(\mathbf{H}), j}$ and $\zeta_{p(\mathbf{H}), k}$ are i.i.d. random variables as long as $j \neq k$. This assumption should be reasonable in practice. For instance, different users within a base station might stay in different locations, and the wireless channels they encounter could have no spatial correlation. Moreover, current base station systems always employ the open-loop power control; hence, the average received SNR of each user at the base station side can be adjusted to an identical value.

Definition 1. In a multi-user scenario, we define $S_{n} \triangleq$ $\sum_{j=1}^{n} \zeta_{p(\mathbf{H}), j}$ as the sum complexity of the ET-FSD of $n$ users, while $\frac{1}{n} S_{n}$ is just the complexity per user.

Obviously, $S_{n}$ and $\frac{1}{n} S_{n}$ are not constants but random variables.

Assumption 1. We assume that, due to the run-time limit, the MIMO receiver is only capable of performing the ET-FSD for $n$ users with the sum complexity being less than $C_{n}$, i.e.,

$$
S_{n}<C_{n}
$$

where $C_{n}$ is called the sum complexity constraint and its subscript $n$ denotes the number of users. Besides, we assume that $n \zeta_{0}<C_{n}<n \zeta_{\bar{p}}$, which means that the sum complexity constraint is less than the worst-case sum complexity $n \zeta_{\bar{p}}$ while larger than $n \zeta_{0}$ that is the minimum possible value of the sum complexity of ET-FSD with $n$ users.

Because the run-time limit is always demanding, we are interested in how the ET-FSD behaves in a multi-user scenario with the sum complexity constraint $C_{n}$ that satisfies $n \zeta_{0}<C_{n}<n \zeta_{\bar{p}}$. Since $n \zeta_{0}<C_{n}<n \zeta_{\bar{p}}$, $S_{n}$ shall exceed $C_{n}$ with certain probability. Once $S_{n} \geq C_{n}$ occurs, to meet the run-time limit, the ET-FSD of some users will have to unconditionally quit before completely executing the tree search of Algorithm 2, which can be seen as a detection outage that would cause performance degradation to the corresponding users. Such detection outage is undesirable; however, it is inevitable. The performance impact brought by the outage event due to the occurrence of $S_{n} \geq$ $C_{n}$ to the ET-FSD of each user will be quantified by means of diversity order loss. The probability $P\left(S_{n} \geq C_{n}\right)$ attracts us because it can reveal the complexity behavior of the ETFSD in a multi-user scenario. To make a fair comparison between the results derived in a multi-user scenario and those traditionally in a single-user scenario, it is better for us to focus the analysis on the behavior of complexity per user. Therefore, we will restrict our attention to studying $P\left(\frac{1}{n} S_{n} \geq \frac{1}{n} C_{n}\right)$.

To conveniently make use of the large deviation principle, the random variables below are introduced

$$
X_{p(\mathbf{H}), j} \triangleq\left\{\begin{array}{ll}
\zeta_{0}, & \text { if } \zeta_{p(\mathbf{H}), j}=\zeta_{0} \\
\zeta_{\bar{p}}, & \text { if } \zeta_{p(\mathbf{H}), j} \neq \zeta_{0}
\end{array},\right.
$$

where $P\left(X_{p(\mathbf{H}), j}=\zeta_{0}\right)=P\left(\eta_{1} \geq \rho^{\gamma}\right)$ and $P\left(X_{p(\mathbf{H}), j}=\right.$ $\left.\zeta_{\bar{p}}\right)=P\left(\zeta_{p(\mathbf{H}), j} \neq \zeta_{0}\right)=P\left(\eta_{1}<\rho^{\gamma}\right)$. Observing that $X_{p(\mathbf{H}), j} \geq \zeta_{p(\mathbf{H}), j}$, we can further define and get

$$
\bar{S}_{n} \triangleq \sum_{j=1}^{n} X_{p(\mathbf{H}), j} \geq S_{n} .
$$

In the following, we will firstly investigate $P\left(\frac{1}{n} \bar{S}_{n} \geq\right.$ $\left.\frac{1}{n} C_{n}\right)$ and then extend to derive an upper bound of $P\left(\frac{1}{n} S_{n} \geq \frac{1}{n} C_{n}\right)$ by using (19). Several theoretical results could be derived as follows.

Lemma 4 (Cramer's theorem for empirical average [29]). Since $X_{p(\mathbf{H}), j}$ satisfies

$$
\varphi(t) \triangleq \mathcal{E}\left(e^{t X_{p(\mathrm{H}), j}}\right)<+\infty, \quad \forall t \in \mathbb{R}
$$

it holds for all $\delta>0$ that

$$
\begin{gathered}
\lim _{n \rightarrow+\infty} \frac{1}{n} \log P\left(\frac{1}{n} \bar{S}_{n} \geq \mathcal{E}\left(X_{p(\mathbf{H}), j}\right)+\delta\right) \\
=-I\left(\mathcal{E}\left(X_{p(\mathbf{H}), j}\right)+\delta\right),
\end{gathered}
$$

where

$$
I(z)=\sup _{t \in \mathbb{R}}(z t-\log \varphi(t)) .
$$


Lemma 5. For $I\left(\mathcal{E}\left(X_{p(\mathbf{H}), j}\right)+\delta\right)$ in (21), its closed-form expression can be given by

$$
\begin{aligned}
I(\mathcal{E}( & \left.\left.X_{p(\mathbf{H}), j}\right)+\delta\right) \\
= & {\left[P\left(A_{1}\right)+\frac{\delta}{\Delta_{\zeta}}\right] } \\
& \times \log \frac{\delta\left[1-P\left(A_{1}\right)\right]+\Delta_{\zeta} P\left(A_{1}\right)\left[1-P\left(A_{1}\right)\right]}{\triangle_{\zeta} P\left(A_{1}\right)\left[1-P\left(A_{1}\right)\right]-\delta P\left(A_{1}\right)} \\
& -\log \frac{\Delta_{\zeta}\left[1-P\left(A_{1}\right)\right]}{\triangle_{\zeta}\left[1-P\left(A_{1}\right)\right]-\delta},
\end{aligned}
$$

where $\Delta_{\zeta} \triangleq \zeta_{\bar{p}}-\zeta_{0}$, and $P\left(A_{1}\right)=1-P\left(X_{p(\mathbf{H}), j}=\zeta_{0}\right)=$ $1-P\left(\zeta_{p(\mathbf{H}), j}=\zeta_{0}\right)$.

\section{Proof. See Appendix 5.}

To make the result of Lemma 5 more comprehensible, we investigate $I\left(\mathcal{E}\left(X_{p(\mathbf{H}), j}\right)+\delta\right)$ in both high-SNR regime. When $\mathrm{SNR} \rho$ grows to $+\infty, P\left(A_{1}\right) \rightarrow 0$ and $1-$ $P\left(A_{1}\right) \rightarrow 1, I\left(\mathcal{E}\left(X_{p(\mathbf{H}), j}\right)+\delta\right)$ can thus be approximated by $I\left(\mathcal{E}\left(X_{p(\mathbf{H}), j}\right)+\delta\right) \approx \frac{\delta}{\Delta_{\zeta}} \log \frac{\delta}{\Delta_{\zeta}-\delta}-\frac{\delta}{\Delta_{\zeta}} \log P\left(A_{1}\right)-$ $\log \left(\frac{\Delta_{\zeta}}{\Delta_{\zeta}-\delta}\right)$. Hence, it is reasonable to conclude that at high SNR, $I\left(\mathcal{E}\left(X_{p(\mathbf{H}), j}\right)+\delta\right)$ is a positive number dominated by $-\delta \log P\left(A_{1}\right) / \triangle_{\zeta}$.

Furthermore, by utilizing Lemma 3 , it can be obtained that $\lim _{\rho \rightarrow+\infty} \frac{I\left(\mathcal{E}\left(X_{p(\mathbf{H}), j}\right)+\delta\right)}{\log \rho} \geq \frac{\delta(1-\gamma)\left(n_{\mathrm{R}}-n_{\mathrm{T}}+1\right)}{\Delta_{\zeta}}$. Assuming that $g(n)$ is a function of $n$, we use the expression $g(n) \asymp e^{-n \gamma}$ with the specific symbol $\asymp$ to represent the mathematic meaning that, for any $\varepsilon>0$, there exists a number $N$ with which $e^{-n(\gamma+\varepsilon)}<g(n)<e^{-n(\gamma-\varepsilon)}$, $\forall n \geq N$.

Lemma 6. From (19) and (21), it follows that $P\left(\frac{1}{n} \bar{S}_{n} \geq \mathcal{E}\left(X_{p(\mathbf{H}), j}\right)+\delta\right) \asymp e^{-n I\left(\mathcal{E}\left(X_{p(\mathbf{H}), j}\right)+\delta\right)}$, where the exact expression of $I\left(\mathcal{E}\left(X_{p(\mathbf{H}), j}\right)+\delta\right)$ is given in Lemma 5 .

For any $\varepsilon>0$, the relationship in Lemma 6 allows us to calculate

$$
\begin{aligned}
\lim _{\rho \rightarrow+\infty} & \frac{n\left[I\left(\mathcal{E}\left(X_{p(\mathbf{H}), j}\right)+\delta\right)-\varepsilon\right]}{\log \rho} \\
& \leq-\lim _{\rho \rightarrow+\infty} \frac{\log P\left(\frac{1}{n} \bar{S}_{n} \geq \mathcal{E}\left(X_{p(\mathbf{H}), j}\right)+\delta\right)}{\log \rho} \\
& \leq \lim _{\rho \rightarrow+\infty} \frac{n\left[I\left(\mathcal{E}\left(X_{p(\mathbf{H}), j}\right)+\delta\right)+\varepsilon\right]}{\log \rho},
\end{aligned}
$$

which further yields

$$
\begin{aligned}
- & \lim _{\rho \rightarrow+\infty} \frac{\log P\left(\frac{1}{n} \bar{S}_{n} \geq \mathcal{E}\left(X_{p(\mathbf{H}), j}\right)+\delta\right)}{\log \rho} \\
= & \lim _{\rho \rightarrow+\infty} \frac{n I\left(\mathcal{E}\left(X_{p(\mathbf{H}), j}\right)+\delta\right)}{\log \rho} \\
\geq & \frac{n \delta(1-\gamma)\left(n_{\mathrm{R}}-n_{\mathrm{T}}+1\right)}{\triangle_{\zeta}} .
\end{aligned}
$$

The above also implies

$$
P\left(\frac{1}{n} \bar{S}_{n} \geq \mathcal{E}\left(X_{p(\mathbf{H}), j}\right)+\delta\right) \dot{\leq} \rho^{-n \delta(1-\gamma)\left(n_{\mathrm{R}}-n_{\mathrm{T}}+1\right) / \Delta_{\zeta}} .
$$

We can observe from Lemma 6 that, as $I\left(\mathcal{E}\left(X_{p(\mathbf{H}), j}\right)+\delta\right)$ is independent of $n$, the larger $n$ is, the smaller the probability $P\left(\frac{1}{n} \bar{S}_{n} \geq \mathcal{E}\left(X_{p(\mathbf{H}), j}\right)+\delta\right)$ will be. Furthermore, given both relations in (19) and (23), it can be obtained that $P\left(\frac{1}{n} S_{n} \geq \mathcal{E}\left(X_{p(\mathbf{H}), j}\right)+\delta\right)$ is upper bounded by $P\left(\frac{1}{n} \bar{S}_{n} \geq \mathcal{E}\left(X_{p(\mathbf{H}), j}\right)+\delta\right)$, and $P\left(\frac{1}{n} S_{n} \geq \mathcal{E}\left(X_{p(\mathbf{H}), j}\right)+\delta\right)$ decays as $n$ increases with speed faster than $\rho^{-n \delta(1-\gamma)\left(n_{\mathrm{R}}-n_{\mathrm{T}}+1\right) / \Delta_{\zeta}}$, i.e.,

$$
P\left(\frac{1}{n} S_{n} \geq \mathcal{E}\left(X_{p(\mathbf{H}), j}\right)+\delta\right) \dot{\leq} \rho^{-n \delta(1-\gamma)\left(n_{\mathrm{R}}-n_{\mathrm{T}}+1\right) / \Delta_{\zeta}}
$$

\subsection{The existence of the scale effect}

Assume that the sum complexity of the SCC-ET-FSD is restricted by the constraint condition in (17). Since the event $\frac{1}{n} S_{n}<\frac{1}{n} C_{n}$ is identical to $S_{n}<C_{n}, \frac{1}{n} C_{n}$ can be regarded as the complexity constraint imposed on every user.

Definition 2. If there exits $C_{n}$ that satisfies $\frac{1}{n} C_{n}<\zeta_{\bar{p}}$, the scale effect is said to exist if the SCC-ET-FSD for each user can maintain full diversity.

It should be stressed here that to prove the existence of the scale effect, we at same time need to think about the sufficient condition for the existence of scale effect regarding $C_{n}$. We are inspired by (24) to assume that $C_{n}$ satisfies

$$
C_{n}^{*}=n \mathcal{E}\left(X_{p(\mathbf{H}), j}\right)+n \delta
$$

Then let us continue to explore the diversity order of the SCC-ET-FSD in the presence of the constraint as in (25). Analogous to Lemma 3, we can get

$$
\lim _{\rho \rightarrow+\infty} \mathcal{E}\left(X_{p(\mathbf{H}), j}\right)=\zeta_{0}
$$


The meaning behind (25) is that the complexity per user, $\frac{1}{n} S_{n}$, would have the worst-case value $\frac{1}{n} C_{n}^{*}=\mathcal{E}\left(X_{p(\mathbf{H}), j}\right)+\delta$ that tends to $\zeta_{0}+\delta$ at an adequately high SNR.

Consequently, let $\mathcal{A}_{n}$ denote the event of $S_{n}$ that violates the constraint of (25) as

$$
\begin{aligned}
\mathcal{A}_{n} & =\left\{S_{n} \mid \frac{1}{n} S_{n} \geq \frac{1}{n} C_{n}^{*}\right\} \\
& =\left\{S_{n} \mid \frac{1}{n} S_{n} \geq \mathcal{E}\left(X_{p(\mathbf{H}), j}\right)+\delta\right\} \\
& =\left\{S_{n} \mid S_{n} \geq C_{n}^{*}\right\},
\end{aligned}
$$

which could lead to the detection outage. Moreover, let $\overline{\mathcal{A}}_{n}$ be the complement of $\mathcal{A}_{n}$ such that $\overline{\mathcal{A}}_{n}=\left\{S_{n}\right\} \backslash \mathcal{A}_{n}$.

It is assumed that, without any sum complexity constraint, $E_{\mathrm{ET}-\mathrm{FSD}, k}$ is the error event of the ET-FSD for the $k$ th user, which satisfies

$$
\begin{aligned}
P\left(E_{\mathrm{ET}-\mathrm{FSD}, k}\right)= & P\left(E_{\mathrm{ET}-\mathrm{FSD}, k} \mid S_{n} \in \mathcal{A}_{n}\right) P\left(S_{n} \in \mathcal{A}_{n}\right) \\
& +P\left(E_{\mathrm{ET}-\mathrm{FSD}, k} \mid S_{n} \in \overline{\mathcal{A}}_{n}\right) P\left(S_{n} \in \overline{\mathcal{A}}_{n}\right) .
\end{aligned}
$$

Next, under the constraint of (25), let $E_{\mathrm{SCC}-\mathrm{ET}-\mathrm{FSD}, k}^{*}$ be the error event of the SCC-ET-FSD for the $k$ th user, which can be expressed as

$$
\begin{aligned}
P\left(E_{\mathrm{SCC}-\mathrm{ET}-\mathrm{FSD}, k}^{*}\right) & \\
= & P\left(E_{\mathrm{SCC}-\mathrm{ET}-\mathrm{FSD}, k}^{*} \mid S_{n} \in \mathcal{A}_{n}\right) P\left(S_{n} \in \mathcal{A}_{n}\right) \\
& \quad+P\left(E_{\mathrm{SCC}-\mathrm{ET}-\mathrm{FSD}, k}^{*} \mid S_{n} \in \overline{\mathcal{A}}_{n}\right) P\left(S_{n} \in \overline{\mathcal{A}}_{n}\right),
\end{aligned}
$$

where the probability $P\left(E_{\mathrm{SCC}-\mathrm{ET}-\mathrm{FSD}, k}^{*} \mid S_{n} \in \mathcal{A}_{n}\right)$ is directly affected by the detection outage.

Lemma 7. Let $d_{E_{\mathrm{SCC}-\mathrm{ET}-\mathrm{FSD}, k}}$ be the diversity order corresponding to $P\left(E_{\mathrm{SCC}-\mathrm{ET}-\mathrm{FSD}, k}^{*}\right)$ such that $P\left(E_{\mathrm{SCC}-\mathrm{ET}-\mathrm{FSD}, k}^{*}\right) \doteq \rho^{-d_{E_{\mathrm{SCC}}^{*} \mathrm{ET}-\mathrm{FSD}, k}}$

It can be obtained that

$$
\begin{array}{r}
\min \left(n_{\mathrm{R}}, \frac{n \delta(1-\gamma)\left(n_{\mathrm{R}}-n_{\mathrm{T}}+1\right)}{\triangle_{\zeta}}\right) \\
\leq d_{E_{\mathrm{SCC}-\mathrm{ET}-\mathrm{FSD}, k}^{*} \leq n_{\mathrm{R}} .}
\end{array}
$$

Proof. See Appendix 6.

Moreover, according to the proof procedure of Lemma 7 , we find that, if $\frac{n \delta(1-\gamma)\left(n_{\mathrm{R}}-n_{\mathrm{T}}+1\right)}{\Delta_{\zeta}} \leq n_{\mathrm{R}}$, the detection outage due to the occurrence of event $S_{n} \geq C_{n}$ might cause diversity order loss to the SCC-ET-FSD for every user; otherwise no loss of diversity order would happen. Therefore, one of the most important results of this study can be obtained as follows.
Theorem 2. We can expect $d_{E_{\mathrm{SCC}-\mathrm{ET}-\mathrm{FSD}, k}^{*}}=n_{\mathrm{R}}$, i.e., the SCC-ET-FSD in the presence of the constraint of (25) maintains full diversity for every user as long as $n$ is sufficiently large such that

$$
n>\frac{n_{\mathrm{R}} \Delta_{\zeta}}{\delta(1-\gamma)\left(n_{\mathrm{R}}-n_{\mathrm{T}}+1\right)} .
$$

Proof. Theorem 2 stems from Lemma 7 in a straightforward manner.

It should be noted here that Theorem 2 shows one sufficient condition for the SCC-ET-FSD to achieve the scale effect, which at same time validates the existence of the scale effect in the SCC-ET-FSD. However, $C_{n}^{*}$ in the form of (25) implicitly varies with $\rho$, which then is neither fixed nor intuitive enough. This motivates us to think of other better sufficient condition for the existence of scale effect with respect to the sum complexity constraint $C_{n}$. Thus, we simply assume that $C_{n}$ satisfies

$$
C_{n}^{\sharp}=n \tau,
$$

where $\tau \in\left(\zeta_{0}, \zeta_{\bar{p}}\right)$ is fixed, regardless of $\rho$ or other system parameters.

Then let $\mathcal{B}_{n}$ denote $\mathcal{B}_{n}=\left\{S_{n} \mid \frac{1}{n} S_{n} \geq \frac{1}{n} C_{n}^{\sharp}\right\}=$ $\left\{S_{n} \mid \frac{1}{n} S_{n} \geq \tau\right\}$, and correspondingly, let $\overline{\mathcal{B}}_{n}$ be the complement of $\mathcal{B}_{n}$, i.e., $\overline{\mathcal{B}}_{n}=\left\{S_{n}\right\} \backslash \mathcal{B}_{n}$.

Hereafter, we would like to let $E_{\mathrm{SCC}-\mathrm{ET}-\mathrm{FSD}, k}^{\sharp}$ be the error event of the SCC-ET-FSD for the $k$ th user under the constraint of (32), which can be written as

$$
\begin{aligned}
& P\left(E_{\mathrm{SCC}-\mathrm{ET}-\mathrm{FSD}, k}^{\sharp}\right) \\
& \quad=P\left(E_{\mathrm{SCC}-\mathrm{ET}-\mathrm{FSD}, k}^{\sharp} \mid S_{n} \in \mathcal{B}_{n}\right) P\left(S_{n} \in \mathcal{B}_{n}\right) \\
& \quad+P\left(E_{\mathrm{SCC}-\mathrm{ET}-\mathrm{FSD}, k}^{\sharp} \mid S_{n} \in \overline{\mathcal{B}}_{n}\right) P\left(S_{n} \in \overline{\mathcal{B}}_{n}\right),
\end{aligned}
$$

where the detection outage impacts $P\left(E_{\mathrm{SCC}-\mathrm{ET}-\mathrm{FSD}, k}^{\sharp}\right)$ by affecting the probability $P\left(E_{\mathrm{SCC}-\mathrm{ET}-\mathrm{FSD}, k}^{\sharp} \mid S_{n} \in \mathcal{B}_{n}\right)$.

By applying the approach of analyzing the vanishing gap to the ML performance developed in [13], the performance gap between the SCC-ET-FSD with the constraint in (32) and the ML detector can be defined in the quantified form as

$$
g_{k}(\rho) \triangleq \frac{P\left(E_{\mathrm{SCC}-\mathrm{ET}-\mathrm{FSD}, k}^{\sharp}\right)}{p_{e \mathrm{ML}}},
$$

where generally $g_{k}(\rho) \geq 1$ since the ML detector has the optimal performance. Furthermore, $g_{k}(\rho)$ has the relation as (34). 
If we take (33) into consideration, let $\delta_{\tau}=\frac{1}{2}\left(\tau-\zeta_{0}\right)$ for any $\tau$ and then let $\mathcal{A}_{n}^{\tau}=\left\{S_{n} \mid \frac{1}{n} S_{n} \geq \mathcal{E}\left(X_{p(\mathbf{H}), j}\right)+\delta_{\tau}\right\}$. It should be clear according to (26) that $\mathcal{E}\left(X_{p(\mathbf{H}), j}\right)+\delta_{\tau}$ tends to be less than $\tau$ eventually with increasing $\rho$. This implies that $\lim _{\rho \rightarrow+\infty} \frac{P\left(S_{n} \in \mathcal{B}_{n}\right)}{p_{e \mathrm{ML}}} \leq \lim _{\rho \rightarrow+\infty} \frac{P\left(S_{n} \in \mathcal{A}_{n}^{\tau}\right)}{p_{e \mathrm{ML}}}$.

$$
\begin{aligned}
g_{k}(\rho)= & \frac{P\left(E_{\mathrm{SCC}-\mathrm{ET}-\mathrm{FSD}, k}^{\sharp} \mid S_{n} \in \mathcal{B}_{n}\right) P\left(S_{n} \in \mathcal{B}_{n}\right)}{p_{e \mathrm{ML}}} \\
& +\frac{P\left(E_{\mathrm{SCC}-\mathrm{ET}-\mathrm{FSD}, k}^{\sharp} \mid S_{n} \in \overline{\mathcal{B}}_{n}\right) P\left(S_{n} \in \overline{\mathcal{B}}_{n}\right)}{p_{e \mathrm{ML}}} \\
\leq & \frac{P\left(S_{n} \in \mathcal{B}_{n}\right)}{p_{e \mathrm{ML}}} \\
& +\frac{P\left(E_{\mathrm{SCC}-\mathrm{ET}-\mathrm{FSD}, k}^{\sharp} \mid S_{n} \in \overline{\mathcal{B}}_{n}\right) P\left(S_{n} \in \overline{\mathcal{B}}_{n}\right)}{p_{e \mathrm{ML}}} .
\end{aligned}
$$

As long as the number of users, $n$, satisfies (31) in which case $P\left(S_{n} \in \mathcal{A}_{n}^{\tau}\right) \dot{<} \rho^{-n_{\mathrm{R}}}$, the above will lead to $\lim _{\rho \rightarrow+\infty} \frac{P\left(S_{n} \in \mathcal{B}_{n}\right)}{p_{\rho}}=0$ since $P\left(S_{n} \in \mathcal{B}_{n}\right)$ tends to zero at a faster rate than $p_{e \mathrm{ML}}$.

On the other hand, similar in essence to (56), it holds that

$$
\begin{aligned}
\lim _{\rho \rightarrow+\infty} \frac{P\left(E_{\mathrm{SCC}-\mathrm{ET}-\mathrm{FSD}, k}^{\sharp} \mid S_{n} \in \overline{\mathcal{B}}_{n}\right) P\left(S_{n} \in \overline{\mathcal{B}}_{n}\right)}{p_{e \mathrm{ML}}} \\
\quad \leq \lim _{\rho \rightarrow+\infty} \frac{P\left(E_{\mathrm{ET}-\mathrm{FSD}, k}\right)}{p_{e \mathrm{ML}}}=1 .
\end{aligned}
$$

Therefore, our derivation would arrive at

$$
\lim _{\rho \rightarrow+\infty} g_{k}(\rho)=1
$$

This means that by following the analytical framework of [13], $P\left(E_{\mathrm{SCC}-\mathrm{ET}-\mathrm{FSD}, k}^{\sharp}\right)$ has a vanishing gap to $p_{e \mathrm{ML}}$. To be more specific, the performance degradation caused by the detection outage due to the occurrence of the event $S_{n} \in \mathcal{B}_{n}$ is insignificant in comparison with the error probability of the ML decoder.

Theorem 3 (Extension of (35)). Given the sum complexity constraint in the form $C_{n}^{\sharp}=n \tau$ (see (32) as well), let $d_{E_{\mathrm{SCC}-\mathrm{ET}-\mathrm{FSD}, k}^{\sharp}}$ denote the diversity order related to $P\left(E_{\mathrm{SCC}-\mathrm{ET}-\mathrm{FSD}, k}^{\sharp}\right)$, i.e., $P\left(E_{\mathrm{SCC}-\mathrm{ET}-\mathrm{FSD}, k}^{\sharp}\right) \doteq$

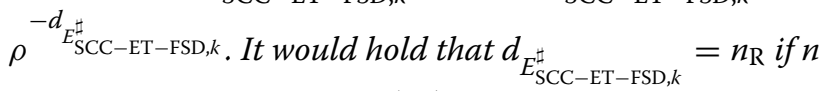
is sufficiently large to meet (31).

Both Theorems 2 and 3 show the existence of the scale effect. Moreover, from Theorem 3, one shall find that if the complexity per user of the SCC-ET-FSD is limited by some fixed $\tau \in\left(\zeta_{0}, \zeta_{\bar{p}}\right)$, since $\zeta_{0}=O(1)$ is polynomial complexity, $\tau$ can be chosen as close as possible to $\zeta_{0}$ such that the complexity constraint per user of the SCC-ET-FSD could have a polynomial ${ }^{\mathrm{d}}$ value while each user of the SCC-ET-FSD still maintains full diversity.

\subsection{The benefit of scale effect}

It is not easy to (theoretically) ensure full-diversity MIMO detection with polynomial complexity, while in a multiuser scenario, we propose to take advantage of the scale effect as a positive factor to improve MIMO detection. How can the scale effect improve MIMO detection? Theorems 2 and 3 give us the answer. Especially, in view of Theorem 2, the diversity order of the SCC-ET-FSD is affected by $n$ as (30). As long as $n$ is large enough to meet (31), the SCC-ET-FSD can maintain full diversity under the constraint of (25), $C_{n}^{*}$, that tends to $n \zeta_{0}+n \delta$ when the average received SNR approaches $+\infty$. Furthermore, in view of Theorem 3, when the sum complexity constraint of the SCC-ET-FSD satisfies $C_{n}^{\sharp}=n \tau$ as (32), the detection outage incurs performance degradation that vanishes considerably fast with increasing $\rho$, in which case the error probabilities of the SCC-ET-FSD for each user and the ML detector have a vanishing gap as (35). Herein, $\tau$ is fixed and can arbitrarily approach $\zeta_{0}$ where $\zeta_{0}=$ $O(1)$ is polynomial. It is also obvious that, under the constraints of both (25) and (32), the scale effect can always be exploited.

\section{Numerical results}

This section is devoted to presenting numerical results that help make the analytic outcomes achieved above more intuitive. Throughout the rest of this section, without loss of generality, we assume that the complexity of a single ET-FSD is $\zeta_{p}$ if the initial number of levels of the FE stage is $p$. Meanwhile, the specification of MIMO system can be referred to Section 1.1 such that the path between each transmitter and receiver antenna suffers Rayleigh fading. Within the Monte Carlo simulation, $10^{6}$ realizations of channel are used.

\subsection{Diversity order of ET-FSD}

The development of the ET-FSD starts the theoretic analysis of the scale effects, where the ET-FSD is proven by Theorem 1 to possess full diversity under the condition specified by line 9 of Algorithm 1. In Figure 2, the error probabilities of the (original) FSD [9] and the ET-FSD are compared with each other under three system deployments. We can observe that there is no evident degradation in performance caused by the introduction of early termination. At this point, it is ensured that the ET-FSD still has full diversity as announced in Theorem 1. 


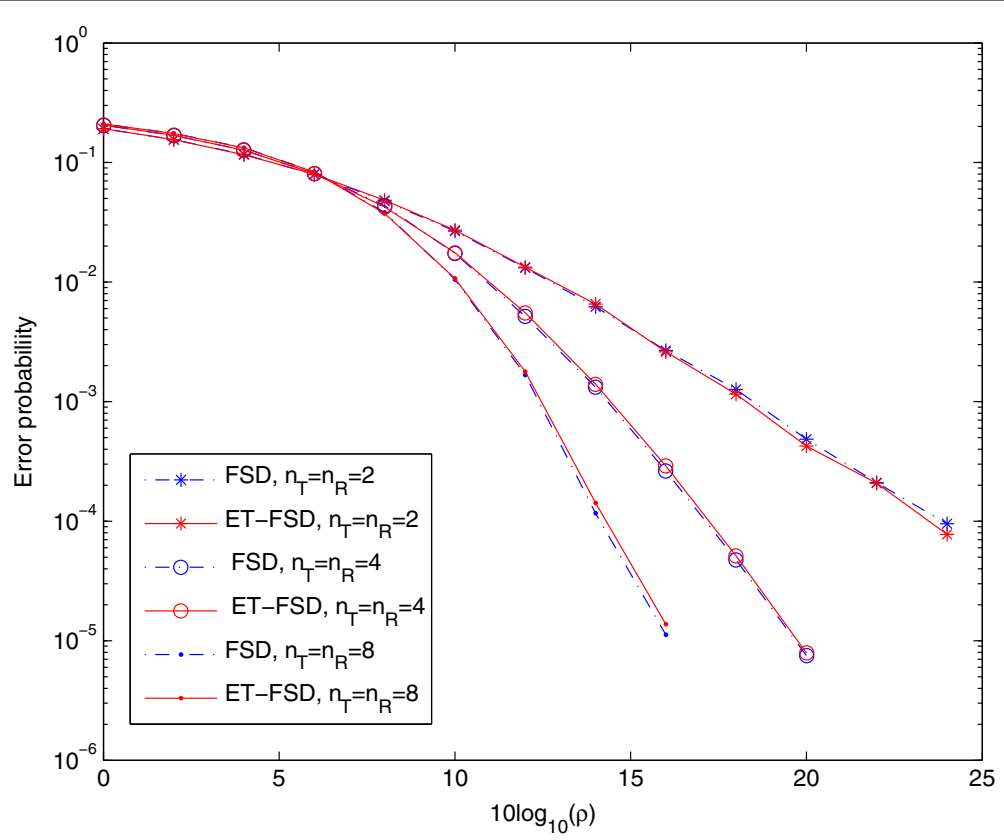

Figure 2 The curves of error probability. The modulation scheme used is QPSK. The number of levels of the FE stage is $p=\left\lceil\sqrt{n_{T}}\right\rceil 1$ for FSD, and the initial number of levels of the FE stage is $\bar{p}=\left\lceil\sqrt{n_{T}}\right\rceil-1$ for ET-FSD. This figure shows the numerical result for Theorem 1.

\subsection{Asymptotic behavior of $P\left(A_{1}\right)$}

According to Lemma 3, $P\left(A_{1}\right)$ tends to 0 as $\rho \rightarrow$ $+\infty$, which decays faster than $\rho^{-(1-\gamma)\left(n_{\mathrm{R}}-n_{\mathrm{T}}+1\right)}$. Several results of this study (including Lemma 6 and Theorems 2 and 3 ) are directly or indirectly affected by the asymptotic behavior of $P\left(A_{1}\right)$. Hence, we pay special attention to the numerical results of $P\left(A_{1}\right)$. In the upper subgraph of Figure 3, the curves of $-10 \log _{10}\left(P\left(A_{1}\right)\right)$ against $10 \log _{10}(\rho)$ are shown for three system deployments, the slopes of which grow large and converge at some constant values as the average SNR $\rho$ increases within the regime 0 to 60 of $10 \log _{10}(\rho)$. Next, we apply the Matlab tools of polynomial curve fitting to acquire the fitting curves for $-10 \log _{10}\left(P\left(A_{1}\right)\right)$ against $10 \log _{10}(\rho)$, as may be seen in the upper subgraph of Figure 3 as well. Then by computing the slopes of the fitting curves via the derivative of the functions associated with the fitting curves, the approximate slopes can be plotted as the lower subgraph of Figure 3, which approach but are not larger than $(1-\gamma)\left(n_{\mathrm{T}}-n_{\mathrm{R}}+\right.$ $1)=1 / 2$. When $-\lim _{\rho \rightarrow+\infty} \frac{\log _{10} P\left(A_{1}\right)}{\log _{10} \rho}$ exists, the slope of the curve of $-10 \log _{10}\left(P\left(A_{1}\right)\right)$ against $10 \log _{10}(\rho)$ shall be asymptotic to $-\frac{\log _{10} P\left(A_{1}\right)}{\log _{10} \rho}$ as $\rho \rightarrow+\infty$. Since in the lower subgraph of Figure 3 the slopes are already shown to converge at a constant value $1 / 2$, it implies that $-\lim _{\rho \rightarrow+\infty} \frac{\log _{10} P\left(A_{1}\right)}{\log _{10} \rho}$ would also converge at $1 / 2$. This is in accordance with what we can expect from Lemma 3.

\subsection{Asymptotic behavior of $\mathcal{E}\left(X_{p(\mathrm{H})}\right)$}

As we can see in (26), $\mathcal{E}\left(X_{p(\mathbf{H}), j}\right) \rightarrow \zeta_{0}$ as $\rho \rightarrow+\infty$. Now it is verified again via Figure 4 that (26) holds true. Given this asymptotic behavior of $\mathcal{E}\left(X_{p(\mathbf{H})}\right)$, we would also be aware that the constraint of sum complexity, $C_{n}^{*}$, which is set as (25), converges at $n \zeta_{0}+$ $n \delta$ at high SNR. In other words, $\frac{1}{n} C_{n}^{*} \rightarrow \zeta_{0}+\delta$ as $\rho \rightarrow+\infty$. Thus, in this case, we can expect that the complexity constraint per user tends to $\zeta_{0}+\delta$ as $\rho$ increases.

\subsection{Statistics of $P\left(\frac{1}{n} \bar{S}_{n} \geq \mathcal{E}\left(X_{p(\mathrm{H}), j}\right)+\delta\right)$}

Based on (19) and (27), it can be found that $P\left(S_{n} \in \mathcal{A}_{n}\right) \leq$ $P\left(\frac{1}{n} \bar{S}_{n} \geq \mathcal{E}\left(X_{p(\mathbf{H}), j}\right)+\delta\right)$, where $P\left(S_{n} \in \mathcal{A}_{n}\right)$ affects the error probability of the SCC-ET-FSD for each user as (29). Lemma 6 reveals the property of $P\left(\frac{1}{n} \bar{S}_{n} \geq \mathcal{E}\left(X_{p(\mathbf{H}), j}\right)+\delta\right)$ which is a key point to prove the existence of the scale effect. Therefore, we present herein the results produced by computer simulations in Figures 5 and 6, where for the purpose of notational simplification, it is defined as $P^{\prime} \triangleq P\left(\frac{1}{n} \bar{S}_{n} \geq \mathcal{E}\left(X_{p(\mathbf{H}), j}\right)+\delta\right)$ and $P^{\prime \prime} \triangleq e^{-n I\left(\mathcal{E}\left(X_{p(\mathbf{H}), j}\right)+\delta\right)}$. In Figure 5 , if $20 \leq 10 \log _{10}(\rho) \leq 90$, the value of $P^{\prime}$ is mostly less than $P^{\prime \prime}$ under the same system configuration with the same $\rho$ since in all the considered system configurations $n_{\mathrm{T}}=n_{\mathrm{R}}$ such that $\Delta \zeta=\zeta_{\bar{p}}-$ $\zeta_{0}$ grows as $n_{\mathrm{R}}$ increases, which might be why we see both $P^{\prime}$ and $P^{\prime \prime}$ of $n_{\mathrm{T}}=n_{\mathrm{R}}=8$ system as the largest among the three systems. In any case, from Figure 5, 

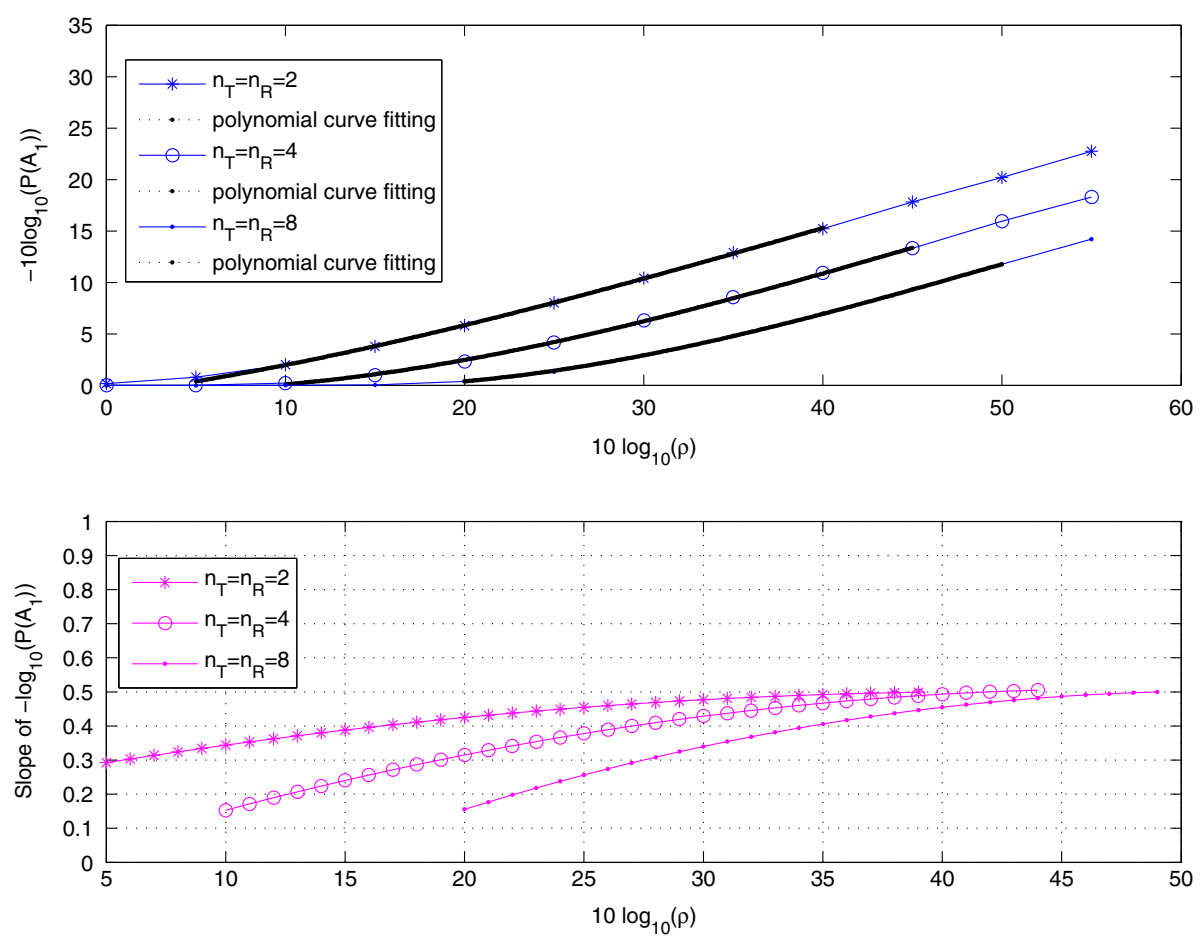

Figure 3 The curves show the asymptotic behavior of $\boldsymbol{P}\left(\boldsymbol{A}_{\mathbf{1}}\right)$ with the event $\boldsymbol{A}_{\mathbf{1}}$ defined as (10). The modulation scheme used is QPSK. $\gamma$ is set to $1 / 2$. The initial number of levels of the FE stage is $\bar{p}=\left\lceil\sqrt{n_{T}}\right\rceil-1$ for ET-FSD. This figure shows the numerical result for Lemma 3 .

$P^{\prime}=P\left(\frac{1}{n} \bar{S}_{n} \geq \mathcal{E}\left(X_{p(\mathbf{H}), j}\right)+\delta\right)$ seems to be less than $P^{\prime \prime}=$ $e^{-n I\left(\mathcal{E}\left(X_{p(\mathbf{H}), j}\right)+\delta\right)}$. In Figure 6 , by setting $n=32,64$, or 160 , the curves of $P^{\prime}=P\left(\frac{1}{n} \bar{S}_{n} \geq \mathcal{E}\left(X_{p(\mathbf{H}), j}\right)+\delta\right), P^{\prime \prime}=$ $e^{-n I\left(\mathcal{E}\left(X_{p(\mathbf{H}), j}\right)+\delta\right)}$, and $e^{-n \delta \log _{e} P\left(A_{1}\right) / \Delta \zeta}$ become closer as $n$ increases. This is coincident with the theoretical results derived earlier.

\section{Conclusion}

This paper provides the insights into the scale effect of ET-FSD. Under the scale effect, the worst-case complexity per user of ET-FSD could decrease as the number of users increases without loss of the diversity order. Within the study, the large deviation principle is used as an

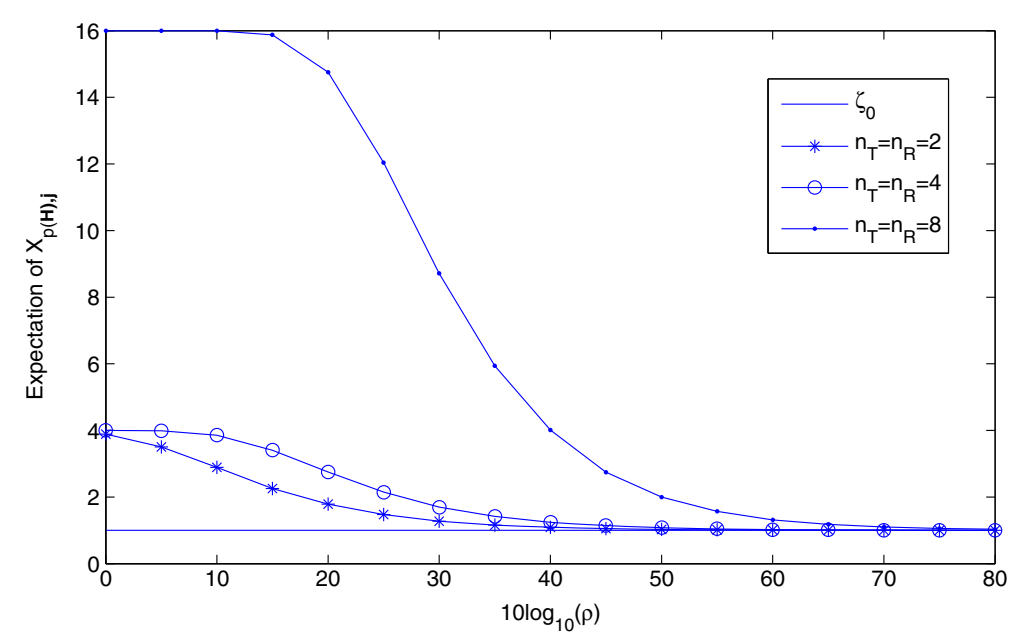

Figure 4 The expectation of $X_{p(\mathbf{H})}, \mathcal{E}\left(X_{\boldsymbol{p}(\mathbf{H})}\right)$ against $10 \log _{\mathbf{1 0}}(\rho)$. The initial number of levels of the FE stage is $\bar{p}=\left\lceil\sqrt{n_{T}}\right\rceil-1$. Note that $X_{p(\mathbf{H}), j}$ is defined in (18). This figure shows the numerical result for (26). 


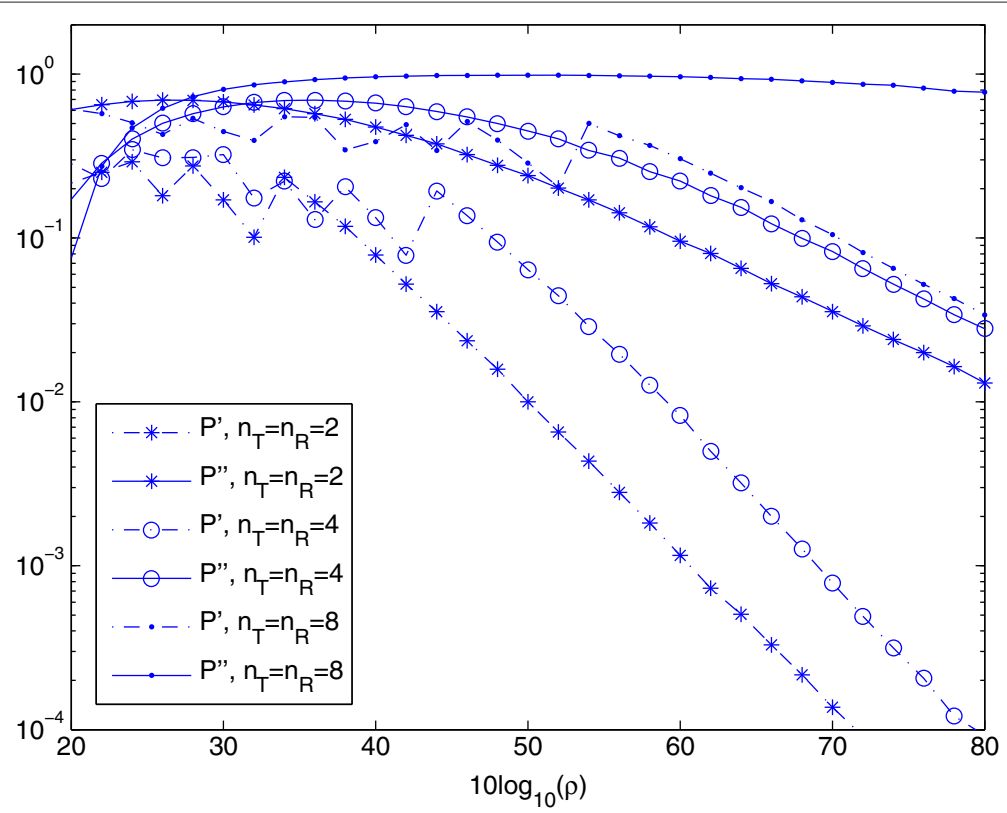

Figure $5 \boldsymbol{P}^{\prime} \triangleq P\left(\frac{1}{n} \bar{S}_{n} \geq \mathcal{E}\left(X_{p(\mathrm{H}), j}\right)+\delta\right)$ and $\boldsymbol{P}^{\prime \prime} \triangleq \boldsymbol{e}^{-\boldsymbol{n} I\left(\mathcal{E}\left(X_{p(\mathrm{H}), j}\right)+\delta\right)}$ against $10 \log _{10}(\rho)$ with $n=16$. The initial number of levels of the FE stage is $\bar{p}=\left\lceil\sqrt{n_{T}}\right\rceil-1$ for ET-FSD.

appropriate and powerful tool to prove the existence of the scale effect.

\section{Endnotes}

a Within this study, unless otherwise stated, the complexity of MIMO detection is measured by the number of all possible candidates of $\mathbf{s}$.

b The polynomial complexity constraint stands for the complexity constraint of MIMO detection being no more than a polynomial function in either $n_{\mathrm{T}}$ or $m$.

c For the multi-user scenario of MIMO system, we assume that all users have the same number of antennas and different users operate on orthogonal resources via time division multiple access or frequency division multiple sccess technologies.

${ }^{d}$ Even if we take the length of s candidate or the complexity of ZF estimate into consideration, the complexity related to $\zeta_{0}$ is still polynomial.

\section{Appendices}

\section{Appendix 1}

\section{Proof of Lemma 2}

Proof. It should be emphasized that, for $0 \leq j \leq p-1$, $A_{0} A_{1} \cdots \bar{A}_{j}$ can represent the event wherein Algorithm 1 encounters early termination in the $j$ th iteration. Meanwhile, the event wherein no early termination happens in Algorithm 1 can be denoted by $A_{0} A_{1} \cdots A_{(p-2)} A_{(p-1)}$. Moreover, the events $A_{0} A_{1} \cdots \bar{A}_{j}$ with $0 \leq j \leq p-1$ and $A_{0} A_{1} \cdots A_{(p-2)} A_{(p-1)}$ are mutually exclusive. Then, it follows from [30] that

$$
\begin{aligned}
p_{e \mathrm{SE}}= & P\left(E_{\mathrm{SE}}, \bar{A}_{0}\right)+P\left(E_{\mathrm{SE}}, A_{0} \bar{A}_{1}\right)+\ldots \\
& +P\left(E_{\mathrm{SE}}, A_{0} A_{1} \cdots A_{(p-2)} \bar{A}_{(p-1)}\right) \\
& +P\left(E_{\mathrm{SE}}, A_{0} A_{1} \cdots A_{(p-2)} A_{(p-1)}\right)
\end{aligned}
$$

and

$$
\begin{aligned}
p_{e \mathrm{ET}-\mathrm{SE}}= & P\left(E_{\mathrm{ET}-\mathrm{SE}}, \bar{A}_{0}\right)+P\left(E_{\mathrm{ET}-\mathrm{SE}}, A_{0} \bar{A}_{1}\right)+\ldots \\
& +P\left(E_{\mathrm{ET}-\mathrm{SE}}, A_{0} A_{1} \cdots A_{(p-2)} \bar{A}_{(p-1)}\right) \\
& +P\left(E_{\mathrm{ET}-\mathrm{SE}}, A_{0} A_{1} \cdots A_{(p-2)} A_{(p-1)}\right)
\end{aligned}
$$

where $P(\cdot, \cdot)$ denotes the joint probability of two joint events.

Concerning the items in (36) and (37), it follows that

$$
\begin{aligned}
& P\left(E_{\mathrm{ET}-\mathrm{SE}}, A_{0} A_{1} \cdots A_{(p-2)} A_{(p-1)}\right) \\
& \quad=P\left(E_{\mathrm{SE}}, A_{0} A_{1} \cdots A_{(p-2)} A_{(p-1)}\right)<p_{e \mathrm{SE}}
\end{aligned}
$$

Given that Proposition 4.2 of Section 2 in [31] states that if one event is contained in another, then the probability 


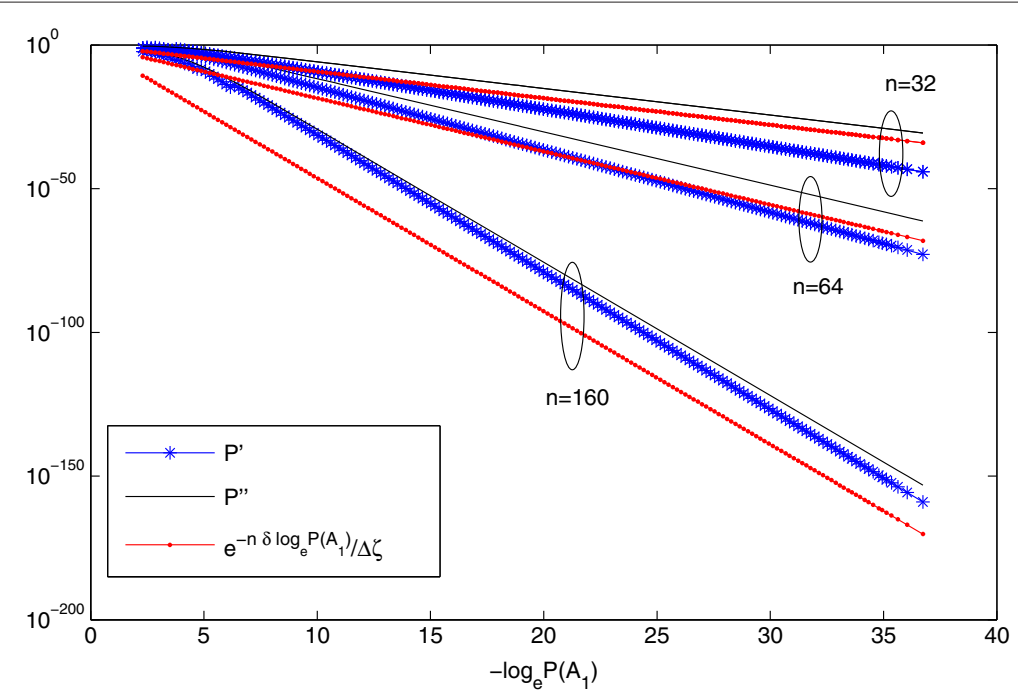

Figure $6 P^{\prime} \triangleq P\left(\frac{1}{n} \bar{S}_{n} \geq \mathcal{E}\left(X_{p(\mathrm{H}), j}\right)+\delta\right), P^{\prime \prime} \triangleq e^{-n I\left(\mathcal{E}\left(X_{p(\mathrm{H}), j}\right)+\delta\right)}$ and $e^{-n \delta \log _{e} P\left(A_{1}\right) / \Delta \zeta}$ against $-\log _{e} \boldsymbol{P}\left(A_{1}\right)$. The initial number of levels of the FE stage is $\bar{p}=\left\lceil\sqrt{n_{T}}\right\rceil-1$ for ET-FSD. This figure shows the numerical result for Lemma 6 .

of the former is no larger than the probability of the latter, (37) leads to

$$
\begin{aligned}
p_{e \mathrm{ET}-\mathrm{SE}} \leq & P\left(E_{\mathrm{ET}-\mathrm{SE}}, \bar{A}_{0}\right)+P\left(E_{\mathrm{ET}-\mathrm{SE}}, \bar{A}_{1}\right)+\ldots \\
& +P\left(E_{\mathrm{ET}-\mathrm{SE}}, \bar{A}_{(p-1)}\right) \\
& +P\left(E_{\mathrm{ET}-\mathrm{SE}}, A_{0} A_{1} \cdots A_{(p-2)} A_{(p-1)}\right)
\end{aligned}
$$

where because of $E_{\mathrm{ET}-\mathrm{SE}}=\bigcup_{i=p+1}^{n_{\mathrm{T}}}\left\{\widetilde{s}_{a_{i}} \neq s_{a_{i}}\right\}$

$$
\begin{aligned}
& P\left(E_{\mathrm{ET}-\mathrm{SE}}, \bar{A}_{j}\right) \\
& \quad \leq \sum_{i=p+1}^{n_{\mathrm{T}}} P\left(\widetilde{s}_{a_{i}} \neq s_{a_{i}}, \eta_{(j+1)} \geq \rho^{\gamma}\right) .
\end{aligned}
$$

In the above, with $p+1 \leq i \leq n_{\mathrm{T}}, \widetilde{s}_{a_{i}}$ is the element of $\widetilde{\mathbf{s}}_{\mathrm{SE}}$, while the corresponding $s_{a_{i}}$ is the element of $\mathbf{s}_{\mathrm{SE}}$.

If $A_{0} A_{1} \cdots A_{(j-1)}$ happens and the ET-FSD meets $\eta_{(j+1)} \geq \rho^{\gamma}$, the event $\bar{A}_{j}$ will occur and $p$ will be set to $j$ by Algorithm 1 before quitting the loop of preprocessing for the SE stage, i.e., $p:=j$. Then with $p+1 \leq i \leq n_{\mathrm{T}}$, let $\eta_{a_{i}}^{\prime}$ denote the post-processing SNR of the ZF estimate for the $a_{i}$ th transmit symbol, $s_{a_{i}}$, in the SE search stage under the condition $\eta_{(j+1)}=x$ with $x \geq \rho^{\gamma}$. In this case, due to the definition of $\eta_{(j+1)}$ as (5), it should be known that $\eta_{(j+1)}=\eta_{(p+1)} \leq \eta_{a_{i}}^{\prime}$. Hence, there exist some $x^{\prime} \geq x$ such that $P\left(\widetilde{s}_{a_{i}} \neq s_{a_{i}} \mid \eta_{(j+1)}=x\right)=P\left(\widetilde{s}_{a_{i}} \neq s_{a_{i}} \mid \eta_{a_{i}}^{\prime}=\right.$ $\left.x^{\prime}\right)$. Herein for each transmit symbol, the ZF estimate in the SE search always utilizes the ML symbol detection. Therefore, it satisfies that

$$
\begin{aligned}
P\left(\widetilde{s}_{a_{i}}\right. & \left.\neq s_{a_{i}} \mid \eta_{(j+1)}=x\right)=P\left(\widetilde{s}_{a_{i}} \neq s_{a_{i}} \mid \eta_{a_{i}}^{\prime}=x^{\prime}\right) \\
& =\bar{N}_{e} Q\left(\sqrt{\frac{x^{\prime} d_{\min }^{2}}{2}}\right) \leq \bar{N}_{e} \exp \left(-\frac{x^{\prime} d_{\min }^{2}}{4}\right) \\
& \leq \bar{N}_{e} \exp \left(-\frac{x d_{\min }^{2}}{4}\right),
\end{aligned}
$$

where $\bar{N}_{e}$ and $d_{\min }$ are the number of nearest neighbors and the minimum distance of separation of the underlying scale constellation, respectively $[1,30]$. To obtain the inequality of (41), the Chernoff bound has been used, i.e., $Q(x) \leq \exp \left(-x^{2} / 2\right)$. Therefore, we can have

$$
\begin{aligned}
P\left(\tilde{s}_{a_{i}} \neq s_{a_{i}}, \eta_{(j+1)} \geq \rho^{\gamma}\right) & \\
& =\int_{\rho^{\gamma}}^{+\infty} P\left(\tilde{s}_{a_{i}} \neq s_{a_{i}} \mid \eta_{(j+1)}=x\right) f_{\eta_{(j+1)}}(x) d x \\
& \leq \bar{N}_{e} \exp \left(-\frac{\rho^{\gamma} d_{\min }^{2}}{4}\right) \int_{\rho^{\gamma}}^{+\infty} f_{\eta_{(j+1)}}(x) d x \\
& \leq \bar{N}_{e} \exp \left(-\frac{\rho^{\gamma} d_{\min }^{2}}{4}\right) .
\end{aligned}
$$

In view of (42), the relation of (40) may also be expressed as

$$
P\left(E_{\mathrm{ET}-\mathrm{SE}}, \bar{A}_{j}\right) \leq\left(n_{\mathrm{T}}-p\right) \bar{N}_{e} \exp \left(-\frac{\rho^{\gamma} d_{\mathrm{min}}^{2}}{4}\right) .
$$


Moreover, the combination of (43) with (38) and (39) leads to

$$
\begin{aligned}
p_{e \mathrm{ET}-\mathrm{SE}} \leq & p\left(n_{\mathrm{T}}-p\right) \bar{N}_{e} \exp \left(-\frac{\rho^{\gamma} d_{\mathrm{min}}^{2}}{4}\right) \\
& +P\left(E_{\mathrm{ET}-\mathrm{SE}}, A_{0} A_{1} \cdots A_{(p-2)} A_{(p-1)}\right) \\
\leq & p\left(n_{\mathrm{T}}-p\right) \bar{N}_{e} \exp \left(-\frac{\rho^{\gamma} d_{\mathrm{min}}^{2}}{4}\right) \\
& +p_{e \mathrm{SE}} .
\end{aligned}
$$

By combining (9) and (44), we can conclude that setting the judging criterion for early termination as (8) is reasonable since $\rho^{\gamma}$ with $0<\gamma<1$ can make the relations in (9) and (44) able to simultaneously establish tight upper bounds (which decay rapidly as $\rho$ increases) for the associated probabilities, $P\left(\eta_{(j+1)}<\rho^{\gamma}\right)$ and $p_{e \mathrm{ET}-\mathrm{SE}}$.

Therefore, Lemma 2 is verified.

\section{Appendix 2}

\section{Background knowledge of diversity order}

Definition 3. Suppose there is a function $f(\rho)$. The symbol $\doteq i s$ always employed to simplify the expressions of exponential equality as $f(\rho) \doteq \rho^{-d} \Leftrightarrow$ $\lim _{\rho \rightarrow+\infty} \frac{\log f(\rho)}{\log \rho}=-d$. If $f(\rho)$ is the error probability of some MIMO detector and $\rho$ denotes average received SNR, $d$ will be the diversity order [27].

Meanwhile, the notation $\dot{\leq}$ and $\dot{<}$ follow after replacing $=$ with $\leq$ and $<$ in above the definition, respectively.

Lemma 8. With the functions $f_{1}(\rho) \doteq \rho^{-d_{1}}$ and $f_{2}(\rho) \doteq$ $\rho^{-d_{2}}$, for $f(\rho)=f_{1}(\rho)+f_{2}(\rho)$, it is established that

$$
f(\rho) \doteq \rho^{-\min \left(d_{1}, d_{2}\right)},
$$

where $\min (\cdot, \cdot)$ returns the minimum of two inputs.

Proof. Without loss of generality, we assume $d_{1} \geq d_{2}$. Thus,

$$
\begin{aligned}
\lim _{\rho \rightarrow+\infty} \frac{\log f(\rho)}{\log \rho}= & \lim _{\rho \rightarrow+\infty} \frac{\log \left(f_{1}(\rho)+f_{2}(\rho)\right)}{\log \rho} \\
= & \lim _{\rho \rightarrow+\infty} \frac{\log f_{2}(\rho)}{\log \rho} \\
& +\lim _{\rho \rightarrow+\infty} \frac{\log \left(1+f_{1}(\rho) / f_{2}(\rho)\right)}{\log \rho} \\
= & -d_{2}=-\min \left(d_{1}, d_{2}\right) .
\end{aligned}
$$

Lemma 9. Having $f_{1}(\rho) \doteq \rho^{-d_{1}}$ and $f_{2}(\rho) \doteq \rho^{-d_{2}}$, if $f_{1}(\rho) \leq f_{2}(\rho)$ for $0<\rho<+\infty$, it will hold true that

$$
d_{1} \geq d_{2}
$$

Proof. We can prove (46) by $d_{1}=-\lim _{\rho \rightarrow+\infty} \frac{\log f_{1}(\rho)}{\log \rho} \geq$ $-\lim _{\rho \rightarrow+\infty} \frac{\log f_{2}(\rho)}{\log \rho}=d_{2}$.

\section{Appendix 3}

\section{Proof of Theorem 1}

Proof. Recalling from (4), keeping (13) in mind and utilizing (45) and (46), we get

$$
\min \left(d_{\mathrm{ML}}, d_{\mathrm{ET}-\mathrm{SE}}\right) \leq d_{\mathrm{ET}-\mathrm{FSD}} \leq d_{\mathrm{ML}}=n_{\mathrm{R}},
$$

where $d_{\mathrm{ET}-\mathrm{FSD}} \leq d_{\mathrm{ML}}$ is true because the ML detector has the optimal performance. Thus, the diversity order of the ET-FSD will not go beyond that of the ML detector.

Once more applying (45) and (46) to (44), the following can be obtained:

$$
d_{\mathrm{ET}-\mathrm{SE}} \geq \min \left(+\infty,-\lim _{\rho \rightarrow+\infty} \frac{\log p_{e \mathrm{SE}}}{\log \rho}\right)
$$

Since $p_{e S E}$ corresponds to the probability of the error event $\widetilde{\mathbf{s}}_{\mathrm{SE}} \neq \mathbf{s}_{\mathrm{SE}}$ in the FSD (or equivalently, the ETFSD without early termination), by referring to [9], we get $-\lim _{\rho \rightarrow+\infty} \frac{\log p_{e S E}}{\log \rho}=\left(n_{\mathrm{R}}-n_{\mathrm{T}}\right)(\bar{p}+1)+(\bar{p}+1)^{2}$. With the special setting of $\bar{p}$ as (3), it is true that $\left(n_{\mathrm{R}}-n_{\mathrm{T}}\right)(\bar{p}+$ 1) $+(\bar{p}+1)^{2} \geq n_{\mathrm{R}}$.

Therefore, (48) yields $d_{\mathrm{ET}-\mathrm{SE}} \geq n_{\mathrm{R}}$, and (47) finally reduces to

$$
d_{\mathrm{ET}-\mathrm{FSD}}=n_{\mathrm{R}}
$$

We can conclude that the ET-FSD is able to achieve full diversity.

\section{Appendix 4}

\section{Proof of Lemma 3}

Proof. For (14), as the event $\zeta_{p(\mathbf{H})}=\zeta_{0}$ is identical to another event $\eta_{1} \geq \rho^{\gamma}$ according to (10), we can get $P\left(\zeta_{p(\mathbf{H})}=\zeta_{0}\right)=P\left(\bar{A}_{1}\right)=1-P\left(A_{1}\right)$. Due to Lemma 1 , $P\left(A_{1}\right) \rightarrow 0$ or $P\left(\bar{A}_{1}\right) \rightarrow 1$ as $\rho \rightarrow+\infty$.

Then taking the logarithm of (9) and then dividing by $\log \rho$, we could derive (15).

For (16), the expectation of $\zeta_{p(\mathbf{H})}$ can be given by

$$
\begin{aligned}
\mathcal{E}\left(\zeta_{p(\mathbf{H})}\right)= & P\left(\bar{A}_{0}\right) \zeta_{0}+P\left(A_{0} \bar{A}_{1}\right) \zeta_{1}+\ldots \\
& +P\left(A_{0} A_{1} \cdots A_{(p-2)} \bar{A}_{(p-1)}\right) \zeta_{(p-1)} \\
& +P\left(A_{0} A_{1} \cdots A_{(p-2)} A_{(p-1)}\right) \zeta_{p} \\
\leq & P\left(\bar{A}_{0}\right) \zeta_{0}+P\left(A_{0}\right) \zeta_{1}+\ldots \\
& +P\left(A_{(p-2)}\right) \zeta_{(p-1)}+P\left(A_{(p-1)}\right) \zeta_{p} .
\end{aligned}
$$

Then by applying Lemma 1, (16) can be verified. This completes the proof of Lemma 3. 


\section{Appendix 5}

\section{Proof of Lemma 5}

Proof. Given the definition of (18) and (20), we get

$$
\varphi(t)=P\left(X_{p(\mathbf{H}), j}=\zeta_{0}\right) e^{t \zeta_{0}}+P\left(X_{p(\mathbf{H}), j}=\zeta_{\bar{p}}\right) e^{t} \zeta_{\bar{p}}
$$

Also, we can have $P\left(X_{p(\mathbf{H}), j}=\zeta_{0}\right)=1-P\left(A_{1}\right)$. As a consequence, (50) becomes

$$
\varphi(t)=\left[1-P\left(A_{1}\right)\right] e^{t \zeta_{0}}+P\left(A_{1}\right) e^{t} \zeta_{\bar{p}}
$$

Define

$$
f(t) \triangleq\left[\mathcal{E}\left(X_{p(\mathbf{H}), j}\right)+\delta\right] t-\log \varphi(t),
$$

where $\mathcal{E}\left(X_{p(\mathbf{H}), j}\right)=\left[1-P\left(A_{1}\right)\right] \zeta_{0}+P\left(A_{1}\right) \zeta_{\bar{p}}$. With the first derivative of $f(t)$, we can compute the critical point of $f(t)$ through the equation as

$$
f^{\prime}(t)=\left[1-P\left(A_{1}\right)\right] \zeta_{0}+P\left(A_{1}\right) \zeta_{\bar{p}}+\delta-\frac{\varphi^{\prime}(t)}{\varphi(t)}=0 .
$$

Incorporating $\varphi(t)$ of (51) into (53) and organizing the items, we have $\delta\left[1-P\left(A_{1}\right)\right]+\delta P\left(A_{1}\right) e^{t\left(\zeta_{\bar{p}}-\zeta_{0}\right)}=$ $-P\left(A_{1}\right)\left[1-P\left(A_{1}\right)\right]\left(\zeta_{\bar{p}}-\zeta_{0}\right)\left(1-e^{t\left(\zeta_{\bar{p}}-\zeta_{0}\right)}\right)$.

Having $\triangle_{\zeta}=\zeta_{\bar{p}}-\zeta_{0}$, the above equation results in $e^{t \Delta \zeta}=$ $\frac{\delta\left[1-P\left(A_{1}\right)\right]+\triangle_{\zeta} P\left(A_{1}\right)\left[1-P\left(A_{1}\right)\right]}{\triangle_{\zeta} P\left(A_{1}\right)\left[1-P\left(A_{1}\right)\right]-\delta P\left(A_{1}\right)}$, from which the critical point of $f(t)$ can be computed as

$$
t_{c p}=\frac{1}{\Delta_{\zeta}} \log \frac{\delta\left[1-P\left(A_{1}\right)\right]+\Delta_{\zeta} P\left(A_{1}\right)\left[1-P\left(A_{1}\right)\right]}{\Delta_{\zeta} P\left(A_{1}\right)\left[1-P\left(A_{1}\right)\right]-\delta P\left(A_{1}\right)}
$$

Investigating the second derivative of $f(t)$, it can be obtained that

$$
\begin{aligned}
f^{\prime \prime}(t) & =-\left(\frac{\varphi^{\prime}(t)}{\varphi(t)}\right)^{\prime}=-\frac{\varphi^{\prime \prime}(t)}{\varphi(t)}+\left(\frac{\varphi^{\prime}(t)}{\varphi(t)}\right)^{2} \\
& =-\frac{P\left(A_{1}\right)\left[1-P\left(A_{1}\right)\right] e^{t\left(\zeta_{0}+\zeta_{\bar{p}}\right)}\left(\zeta_{0}-\zeta_{\bar{p}}\right)^{2}}{(\varphi(t))^{2}}<0 .
\end{aligned}
$$

Hence, it is apparent that the critical point obtained by (54) yields the maximum value of $f(t)$, which can be written as (55).

$$
\begin{aligned}
f_{\max }=f & \left(\frac{1}{\triangle_{\zeta}} \log \frac{\delta\left[1-P\left(A_{1}\right)\right]+\triangle_{\zeta} P\left(A_{1}\right)\left[1-P\left(A_{1}\right)\right]}{\triangle_{\zeta} P\left(A_{1}\right)\left[1-P\left(A_{1}\right)\right]-\delta P\left(A_{1}\right)}\right) \\
=[1 & \left.-P\left(A_{1}\right)\right] \zeta_{0} t+P\left(A_{1}\right) \zeta_{\bar{p}} t \\
& +\delta t-\zeta_{0} t-\log \left(\left[1-P\left(A_{1}\right)\right]\right. \\
& \left.+P\left(A_{1}\right) e^{t \Delta_{\zeta}}\right)\left.\right|_{t=}\left(\frac{1}{\Delta_{\zeta}} \log \frac{\delta\left[1-P\left(A_{1}\right)\right]+\Delta_{\zeta} P\left(A_{1}\right)\left[1-P\left(A_{1}\right)\right]}{\Delta_{\zeta} P\left(A_{1}\right)\left[1-P\left(A_{1}\right)\right]-\delta P\left(A_{1}\right)}\right)
\end{aligned}
$$

$$
\begin{aligned}
=[ & \left.P\left(A_{1}\right)+\frac{\delta}{\triangle_{\zeta}}\right] \\
& \times \log \frac{\delta\left[1-P\left(A_{1}\right)\right]+\triangle_{\zeta} P\left(A_{1}\right)\left[1-P\left(A_{1}\right)\right]}{\triangle_{\zeta} P\left(A_{1}\right)\left[1-P\left(A_{1}\right)\right]-\delta P\left(A_{1}\right)} \\
& -\log \left(\frac{\triangle_{\zeta}\left[1-P\left(A_{1}\right)\right]}{\triangle_{\zeta}\left[1-P\left(A_{1}\right)\right]-\delta}\right) .
\end{aligned}
$$

It should be clear from a look at the items on the right-hand side of the second equality in (55) that [1 $\left.P\left(A_{1}\right)\right] \zeta_{0} t+P\left(A_{1}\right) \zeta_{\bar{p}} t+\delta t=P\left(A_{1}\right)\left(\zeta_{\bar{p}}-\zeta_{0}\right) t+\zeta_{0} t+\delta t$.

It can also be observed that $\log \left(\left[1-P\left(A_{1}\right)\right]+P\left(A_{1}\right)\right.$ $\left.e^{t \Delta_{\zeta}}\right)=\log \left(1+P\left(A_{1}\right)\left(e^{t \Delta_{\zeta}}-1\right)\right)$.

By replacing $t$ with the critical point of (54), we get $\log \left(\left[1-P\left(A_{1}\right)\right]+P\left(A_{1}\right) e^{t_{c p} \Delta_{\zeta}}\right)=\log \left(\frac{\Delta_{\zeta}\left[1-P\left(A_{1}\right)\right]}{\Delta_{\zeta}\left[1-P\left(A_{1}\right)\right]-\delta}\right)$.

Therefore, the exact expression of the maximum value of $f(t)$, i.e., $f_{\max }$, is eventually obtained by (55). By recalling (22), (52), and (55), we obtain $I\left(\mathcal{E}\left(X_{p(\mathbf{H}), j}\right)+\delta\right)=f_{\text {max }}$.

Thus, the validation of Lemma 5 is established.

\section{Appendix 6}

\section{Proof of Lemma 7}

Proof. Based on the conclusion of (49), it follows that $P\left(E_{\mathrm{ET}-\mathrm{FSD}, k}\right) \doteq \rho^{-n_{\mathrm{R}}}$.

Then in view of (28), (45), and (46), it suffices to show that $P\left(E_{\mathrm{ET}-\mathrm{FSD}, k} \mid S_{n} \in \overline{\mathcal{A}}_{n}\right) P\left(S_{n} \in \overline{\mathcal{A}}_{n}\right) \dot{\leq} \rho^{-n_{\mathrm{R}}}$.

Provided that, when $S_{n} \in \overline{\mathcal{A}}_{n}$, no detection outage occurs, which means that the sum complexity constraint has no effect on the detected result, it is true that $P\left(E_{\mathrm{ET}-\mathrm{FSD}, k} \mid S_{n} \in \overline{\mathcal{A}}_{n}\right) P\left(S_{n} \in \overline{\mathcal{A}}_{n}\right)=$ $P\left(E_{\mathrm{SCC}-\mathrm{ET}-\mathrm{FSD}, k}^{*} \mid S_{n} \in \overline{\mathcal{A}}_{n}\right) P\left(S_{n} \in \overline{\mathcal{A}}_{n}\right)$.

Thus,

$$
P\left(E_{\mathrm{SCC}-\mathrm{ET}-\mathrm{FSD}, k}^{*} \mid S_{n} \in \overline{\mathcal{A}}_{n}\right) P\left(S_{n} \in \overline{\mathcal{A}}_{n}\right) \dot{\leq} \rho^{-n_{\mathrm{R}}} .
$$

For $P\left(S_{n} \in \mathcal{A}_{n}\right)$, by referring to (19), we can get

$$
\begin{aligned}
P\left(S_{n} \in \mathcal{A}_{n}\right) & =P\left(\frac{1}{n} S_{n} \geq \mathcal{E}\left(X_{p(\mathbf{H}), j}\right)+\delta\right) \\
& \leq P\left(\frac{1}{n} \bar{S}_{n} \geq \mathcal{E}\left(X_{p(\mathbf{H}), j}\right)+\delta\right) .
\end{aligned}
$$

As $P\left(E_{\mathrm{SCC}-\mathrm{ET}-\mathrm{FSD}, k}^{*} \mid S_{n} \in \mathcal{A}_{n}\right) \leq 1$, together with (23) and (57), it is established that

$$
\begin{aligned}
P( & \left.E_{\mathrm{SCC}-\mathrm{ET}-\mathrm{FSD}, k}^{*} \mid S_{n} \in \mathcal{A}_{n}\right) P\left(S_{n} \in \mathcal{A}_{n}\right) \\
& \dot{\leq} P\left(S_{n} \in \mathcal{A}_{n}\right) \dot{\leq} P\left(\frac{1}{n} \bar{S}_{n} \geq \mathcal{E}\left(X_{p(\mathbf{H}), j}\right)+\delta\right) \\
& \dot{\leq} \rho^{-n \delta(1-\gamma)\left(n_{\mathrm{R}}-n_{\mathrm{T}}+1\right) / \Delta_{\zeta}} .
\end{aligned}
$$

Therefore, by combining (56) with (58) while having (45), the proof of Lemma 7 is complete. 


\section{Competing interests}

The authors declare that they have no competing interests.

\section{Acknowledgements}

The authors wish to thank the anonymous reviewers for their helpful comments and their thoughtful reviews. They greatly improved the quality of the paper. A short version of this paper was presented at the IEEE PIMRC 2012. This work is sponsored by the BUPT Innovative Funds for Young Scholars (2012RC0507 and 2013RC0210), National Science and Technology Major Projects under grants 2012ZX03003011, 2012ZX03003007 and 2013ZX03003012, National Natural Science Foundation of China under grant nos. 60572120, 61001117, and 60602058, the National Key Basic Research Program of China (973 Program) under grant no. 2009CB320400, and the Joint Funds of NSFC-Guangdong under grant U1035001.

\section{Author details}

${ }^{1}$ Automation School, Beijing University of Posts and Telecommunications (BUPT), Beijing 100876, China. ${ }^{2}$ Electronic School, Beijing University of Posts and Telecommunications (BUPT), Beijing 100876, China. ${ }^{3}$ Wireless Signal Processing and Network Lab (Key Lab. of Universal Wireless Communication, Ministry of Education), Beijing University of Posts and Telecommunications (BUPT), Beijing 100876, China.

\section{Received: 8 January 2013 Accepted: 12 June 2013}

\section{Published: 3 July 2013}

\section{References}

1. A Paulraj, R Nabar, D Gore, Introduction to Space-Time Wireless Communications, 1st edn. (Cambridge University Press, Cambridge, 2003)

2. U Fincke, M Pohst, Improved methods for calculating vectors of short length in a lattice, including a complexity analysis. Math. Comp. 44, 463-471 (1985)

3. E Viterbo, J Boutros, A universal lattice code decoder for fading channels. IEEE Trans. Inform. Theory. 45(5), 1639-1642 (1999)

4. E Agrell, T Eriksson, A Vardy, K Zeger, Closest point search in lattice. IEEE Trans. Inform. Theory. 48(8), 2201-2214 (2002)

5. B Hassibi, H Vikalo, On the sphere-decoding algorithm I. Expected complexity. IEEE Trans. Signal Process. 53, 2806-2818 (2005)

6. A Wiesel, YC Eldar, S Shamai, Semidefinite relaxation for detection of 16-QAM signaling in MIMO channels. IEEE Signal Process. Lett. 12(9), 653-656 (2005)

7. KLee, J Chun, ML symbol detection based on the shortest path algorithm for MIMO systems. IEEE Trans. Signal Process. 55(11), 5477-5484 (2007)

8. Z Guo, P Nilsson, Algorithm and implementation of the K-best sphere decoding for MIMO detection. IEEE J. Select. Areas Commun. 24(3), 491-503 (2006)

9. LG Barbero, JS Thompson, in Proceedings of IEEE International Conference on Acoustics, Speech and Signal Processing 2006. Performance analysis of a fixed-complexity sphere decoder in high-dimensional MIMO systems (IEEE New York, 2006), pp. 14-19

10. B Gestner, X Ma, DV Anderson, Incremental lattice reduction: motivation, theory, and practical implementation. IEEE Trans. Wireless. Communi. 11(1), 188-198 (2012)

11. D Wubben, D Seethaler, J Jalden, G Matz, Lattice reduction. IEEE Trans. Wireless. Communi. 28(3), 70-91 (2011)

12. JW Choi, B Shim, AC Singer, NI Cho, Low-complexity decoding via reduced dimension maximum-likelihood search. IEEE Trans. Signal Process. 58(3), 1780-1793 (2010)

13. J Jalden, P Elia, Sphere decoding complexity exponent for decoding full rate codes over the quasi-static MIMO channel. IEEE Trans. Inform. Theory. 58, 5785-5803 (2012)

14. W Abediseid, M Damen, Lattice sequential decoder for coded MIMO channel: performance and complexity analysis. IEEE Trans. Inform. Theory (2010). doi:10.1109/ISIT.2010.5513497

15. J Goldberger, A Leshem, MIMO detection for high-order QAM based on a Gaussian tree approximation. IEEE Trans. Inform. Theory. 57(8), 4973-4982 (2011)

16. J Jalden, B Ottersten, On the complexity of sphere decoding in digital communications. IEEE Trans. Signal Process. 53(4), 1474-1484 (2005)
17. J Jalden, LG Barbero, B Ottersten, JS Thompson, The error probability of the fixed-complexity sphere decoder. IEEE Trans. Signal Process. 57(7), 2711-2720 (2009)

18. J Jalden, B Ottersten, in Proceedings of International Symposium on Information Theory 2005. On the limits of sphere decoding (IEEE New York, 2005), pp. 1691-1695

19. M Taherzadeh, AK Khandani, On the limitations of the naive lattice decoding. IEEE Trans. Inform. Theory. 56(10), 4820-4826 (2010)

20. R Qian, Y Qi, T Peng, W Wang, J Yang, On the scale effects oriented MIMO detector: diversity order, worst-case unit complexity and scale effects. Elsevier Signal Process. 93(1), 277-287 (2013)

21. R Qian, Y Qi, T Peng, W Wang, in Proceedings of the IEEE Globecom 2012 Workshop on Cloud Base-Station and Large-Scale Cooperative Communications. Exploiting scalar effects of MIMO detection in cloud base-station: feasible scheme and universal significance (IEEE New York, 2012), pp. 216-221

22. J Jalden, L Barbero G, B Ottersten, JS Thompson, in Proceedings of IEEE International Conference on Acoustics, Speech and Signal Processin 2007. Full diversity detection in MIMO systems with a fixed-complexity sphere decoder (IEEE New York, 2007), pp. III-49

23. D Gore, RW Heath Jr., A Paulraj, in Proceedings of the IEEE International Symposium on Information Theory 2002. On performance of the zero forcing receiver in presence of transmit correlation (IEEE New York, 2002), p. 159

24. R Narasimhan, Spatial multiplexing with transmit antenna and constellation selection for correlated MIMO fading channels. IEEE Trans. Signal Process. 51(11), 2829-2838 (2003)

25. H Lutkepohl, Handbook of Matrices. (Wiley, Chichester, 1996)

26. A Khoshnevis, A Sabharwal, in Proceedings of the Allerton Conference on Communication, Control and Computing. On diversity and multiplexing gain of multiple antenna systems with transmitter channel information (University of Illinois Monticello, 2004)

27. L Zheng, D Tse, Diversity and multiplexing: a fundamental tradeoff in multiple-antenna channels. IEEE Trans. Inform. Theory. 49(5), 1073-1096 (2003)

28. Economies of scale. (Wikipedia, 2013). http://en.wikipedia.org/wiki/ Economies of scale. Accessed 1 Feb 2013

29. F Hollander, Large Deviations. (American Mathematical Society, Providence, 2008)

30. J Proakis G, Digital Communications, 4th edn. (McGraw-Hill, New York, 1995)

31. SM Ross, A First Course in Probability, 8th edn. (Pearson Education, Upper Saddle River, 2009)

doi:10.1186/1687-6180-2013-125

Cite this article as: Qian et al: Scale effect analysis of early-termination fixed-complexity sphere detector. EURASIP Journal on Advances in Signal Processing 2013 2013:125.

\section{Submit your manuscript to a SpringerOpen ${ }^{\circ}$ journal and benefit from:}

- Convenient online submission

Rigorous peer review

- Immediate publication on acceptance

- Open access: articles freely available online

- High visibility within the field

- Retaining the copyright to your article

Submit your next manuscript at $>$ springeropen.com 